\title{
On the kinematic evolution of young local associations and the Scorpius-Centaurus complex ${ }^{\star}$
}

\author{
D. Fernández ${ }^{1,2, \star \star}$, F. Figueras ${ }^{1}$, and J. Torra ${ }^{1}$ \\ 1 Departament d'Astronomia i Meteorologia, IEEC-Universitat de Barcelona, Av. Diagonal 647, 08028 Barcelona, Spain \\ e-mail: david.fernandez@am.ub.es \\ 2 Observatori Astronòmic del Montsec, Consorci del Montsec, Plaça Major 1, 25691 Àger (Lleida), Spain
}

Received 25 April 2007 / Accepted 13 December 2007

\section{ABSTRACT}

\begin{abstract}
Context. Over the last decade, several groups of young (mainly low-mass) stars have been discovered in the solar neighbourhood (closer than $\sim 100 \mathrm{pc}$ ), thanks to cross-correlation between X-ray, optical spectroscopy and kinematic data. These young local associations - including an important fraction whose members are Hipparcos stars - offer insights into the star formation process in low-density environments, shed light on the substellar domain, and could have played an important role in the recent history of the local interstellar medium.

Aims. To study the kinematic evolution of young local associations and their relation to other young stellar groups and structures in the local interstellar medium, thus casting new light on recent star formation processes in the solar neighbourhood.

Methods. We compiled the data published in the literature for young local associations. Using a realistic Galactic potential we integrated the orbits for these associations and the Sco-Cen complex back in time.

Results. Combining these data with the spatial structure of the Local Bubble and the spiral structure of the Galaxy, we propose a recent history of star formation in the solar neighbourhood. We suggest that both the Sco-Cen complex and young local associations originated as a result of the impact of the inner spiral arm shock wave against a giant molecular cloud. The core of the giant molecular cloud formed the Sco-Cen complex, and some small cloudlets in a halo around the giant molecular cloud formed young local associations several million years later. We also propose a supernova in young local associations a few million years ago as the most likely candidate to have reheated the Local Bubble to its present temperature.
\end{abstract}

Key words. Galaxy: kinematics and dynamics - Galaxy: solar neighborhood - Galaxy: open clusters and associations: general stars: kinematics - stars: formation - ISM: individual objects: Local Bubble

\section{Introduction}

The first moving groups of stars were discovered early on in the history of Galactic dynamics. As early as 1869 , Proctor published a work in which he identified a group of stars around the Hyades open cluster that was moving together through the Galaxy. He also found another 5 comoving stars in the Ursa Major constellation. In the 1960s, Eggen suggested the existence of a Local Association (see for example Eggen 1961, 1965a,b) formed by a group of young stars with approximately the same spatial velocity (also referred as the Pleiades moving group). Eggen's Local Association included the nearest bright B-type stars, stars in the Pleiades, the $\alpha$ Perseus and IC2602 clusters, and the stars belonging to the Sco-Cen complex. However, it was difficult to defend a unitary view of the group due to the wide range of ages ( $\$ 100 \mathrm{Myr})$ and widespread spatial distribution $(\sim 300 \mathrm{pc})$ of its constituent stars.

At the same time as Eggen was studying his Local Association, the first X-ray detectors were installed in rockets and launched into space. This led to the discovery of the diffuse soft X-ray background (SXRB). The anticorrelation observed between the SXRB and the HI column density was rapidly interpreted as evidence of a local cavity in the interstellar

\footnotetext{
* Tables 7-14 are only available in electronic form at http://www . aanda.org

$\star \star$ At present at Institut d'Estudis Espacials de Catalunya (IEEC), c/. Gran Capità 2-4, 08034 Barcelona, Spain.
}

medium (ISM) filled by an X-ray emitting plasma, which became known as the Local Bubble (LB). The launch of the ROSAT satellite in 1990 allowed the LB to be studied in more detail. The opening of the X-ray window in the sky and observations of stars belonging to clusters with known ages also led to the realisation that X-ray emission persists in young stars for a period of the order of $100 \mathrm{Myr}$. An important fraction of these young, X-ray emitting stars are $\mathrm{T}$ Tauri stars. These very young ( $\$ 10 \mathrm{Myr}$ ) stars also exhibit excess IR emission (due to the presence of nearby heated dust particles forming disks or envelopes) as well as UV line and continuum emission produced by the accretion of the surrounding gas and dust. When the stars reach the age of $\sim 10$ Myr, both IR emissions and optical activity decline considerably. X-ray activity then becomes the basis for ascertaining youthfulness.

The ROSAT All-Sky Survey (RASS) detected more than $100000 \mathrm{X}$-ray sources at $\mathrm{keV}$ energies (Voges et al. 2000). Using a kinematic approach and searching for groups of comoving stars allows us to determine which of them are $\mathrm{T}$ Tauri stars. This greatly reduces the number of candidate stars that must be observed spectroscopically to confirm their youthfulness.

In this way, several young stellar associations have been discovered within $100 \mathrm{pc}$ of Earth during the last decade (see Jayawardhana 2000; and Zuckerman \& Song 2004, for a recent review). The stars belonging to these young local associations (YLA) have ages ranging from a few million to several tens of millions of years. Due to their proximity to us, these stars are 
spread over a large area of the sky (up to several hundred square degrees) making it difficult to identify the associations as single entities. Furthermore, they are far away from molecular clouds or star forming regions (SFR). This is why they remained unnoticed until recently.

These young stars offer insights into the star formation process in low-density environments, which is different from the dominant mechanism observed in denser SFR. The discovery of these YLA has also contributed to substellar astrophysics, since dozens of dwarfs have been identified in them. For instance, it has been confirmed that isolated brown dwarfs can form in these low-density environments (see for example Webb et al. 1999; Lowrance et al. 1999; Chauvin et al. 2003). YLA also provide important clues regarding recent star formation in the solar neighbourhood (since they contain the youngest stars near the Sun) and its effect on the local ISM.

In Sect. 2 of this paper we review current understanding of the LB and the Sco-Cen complex and present published data for the nearly 300 stars belonging to YLA. In Sect. 3 we present our method for orbit integration, which makes use of a Galactic potential that includes the general axisymmetric potential and the perturbations due to the Galaxy's spiral structure and the central bar. This leads us to study, in Sect. 4, the origin and evolution of the local structures and, in Sect. 5, to propose a scenario for recent star formation in the solar neighbourhood. This scenario explains the origin of the Sco-Cen complex and YLA through the collision of a parent giant molecular cloud (GMC) with the Sagittarius-Carina spiral arm. Finally, the conclusions of our work are summarised in Sect. 6.

\section{The nearest solar neighbourhood}

In this section we consider three important structures: the Local Bubble (its spatial structure is presented); the Sco-Cen (-LupusCrux) complex (spatial structure, kinematics and age are presented); and the recently discovered YLA (for which we have produced a complete compendium of their members and mean properties).

\subsection{The Local Bubble}

Locally, within the nearest $100 \mathrm{pc}$, the ISM is dominated by the LB. The displacement model (Snowden et al. 1990, 1998) for this structure assumes that the irregular local HI cavity is filled by an X-ray-emitting plasma, with an emission temperature of $\sim 10^{6} \mathrm{~K}$ and a density, $n_{\mathrm{e}}$, of $\sim 0.005 \mathrm{~cm}^{-3}$. Using the distance to the MBM 12 molecular cloud as a distance scale, Snowden et al. (1998) derived an extension for the LB of 40 to $130 \mathrm{pc}$, it being larger at higher Galactic latitudes and smaller nearer the equator. Nevertheless, there is no agreement in the literature concerning the distance to MBM 12; values range from 60 to 300 pc (see for example Hearty et al. 2000a,b; Luhman 2001; Andersson et al. 2002). This could explain why the extension of the LB calculated from SXRB observations does not agree (by a few tens of parsecs) with the extension of the local cavity derived from HI observations (Paresce 1984; Welsh et al. 1994).

Sfeir et al. (1999) and Lallement et al. (2003) obtained the contours of the LB from $\mathrm{NaI}$ absorption measurements taken towards a selected set of stellar targets with Hipparcos parallaxes of up to $350 \mathrm{pc}$ from the Sun. These observations allowed the authors to draw maps of the neutral gas distribution in the local ISM and, in particular, to trace the contours and extension of the LB with an estimated precision of $\approx \pm 20 \mathrm{pc}$ in most directions.

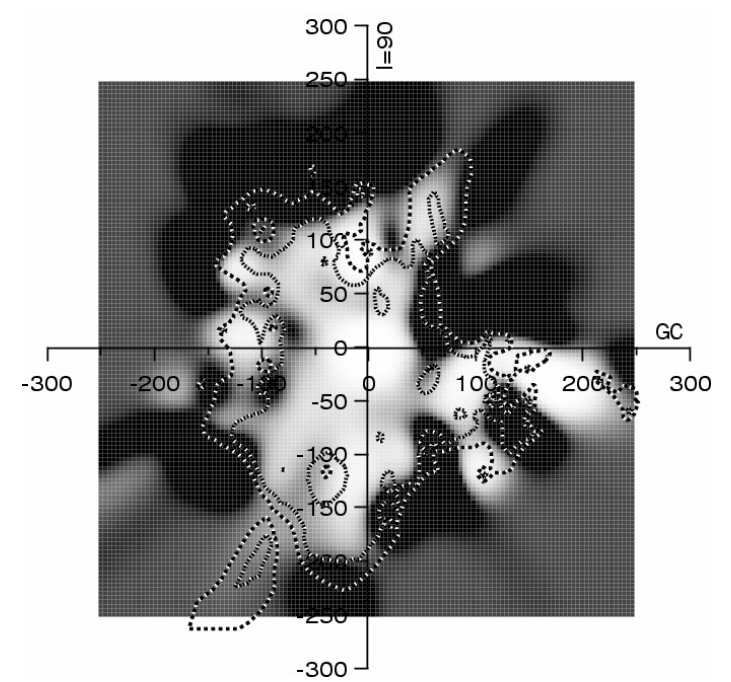

Fig. 1. 3D volumic density of the Local Bubble from the iso equivalent width contours for $W=20 \mathrm{~m} \AA$ and $50 \mathrm{~m} \AA$ (from Lallement et al. 2003).

Lallement et al. (2003) concluded that for heliocentric distances of about 70-90 pc, the equivalent width of the NaI D-line doublet increases from $<5 \mathrm{~m} \AA$ to $>30 \mathrm{~m} \AA$. They interpret this sudden increase as a neutral gas boundary of the local cavity, i.e. the LB limit. This boundary (see Fig. 1) has some interstellar tunnels that connect the LB with other cavities, such as Loop I and the supershell GSH238+00+09. In the meridian plane, the structure is clearly observed to tilt by about $20^{\circ}$ (see Fig. 5 in Lallement et al. 2003). This tilt is probably the result of squeezing of the LB by the surrounding structures belonging to the Gould Belt, which form a local chimney perpendicular to the plane of the Belt. (Sfeir et al. 1999; Welsh et al. 1999).

Some LB formation models have been proposed (see for example Cox 1998), most of which involve several recent supernovae (SNe). The Sco-Cen complex is the most likely candidate to have sheltered these SNe (Maíz-Apellániz 2001; Fuchs et al. 2006).

\subsection{The Sco-Cen complex}

The Sco-Cen complex dominates the fourth Galactic quadrant (de Zeeuw et al. 1999, hereafter Z99). It is a region of recent star formation and contains an important fraction of the most massive stars in the solar neighbourhood. In the 1960s the complex was split into three components (Blaauw 1960): Upper Scorpius (US), Upper Centaurus Lupus (UCL) and Lower Centaurus Crux (LCC). The ISM related to this complex was studied by de Geus (1992), who found that there is not much interstellar material associated with UCL and LCC, whereas filamentous structures connect US with the cloud complex in Ophiuchus. The most widely accepted ages for the components of the complex were derived by de Geus et al. (1989) from isochrones in the HR diagram: 5-6 Myr for US, 14-15 Myr for UCL and 11-12 Myr for LCC. Blaauw $(1964,1991)$ suggested that this age progression was the result of a sequence of star formation events in the GMC that formed all of the Sco-Cen region. These classical ages have recently been questioned by studies of the lowmass component of the complex. The ages obtained from such studies are in the range of 8-10 Myr for US and 16-20 Myr for UCL and LCC (Sartori et al. 2001; Mamajek et al. 2002; Sartori et al. 2003, hereafter S03). However, Preibisch et al. (2002) once again obtained an age of $~ 5 \mathrm{Myr}$ for US from the HR diagram of 
Table 1. Mean spatial coordinates and heliocentric velocity components of the young local associations and the Sco-Cen complex (in the latter case, data from Z99, M02 and S03). In brackets, the standard deviation of the sampling distribution. $N$ is the number of known members in each association $\left(N_{\mathrm{r}}\right.$ with distance determination and $N_{\mathrm{k}}$ with complete kinematic data).

\begin{tabular}{|c|c|c|c|c|c|c|c|c|c|c|c|c|}
\hline \multicolumn{2}{|c|}{ Association } & $\begin{array}{r}\overline{\xi^{\prime}} \\
(\mathrm{pc})\end{array}$ & $\begin{array}{r}\overline{\eta^{\prime}} \\
(\mathrm{pc})\end{array}$ & $\begin{array}{r}\overline{\zeta^{\prime}} \\
(\mathrm{pc})\end{array}$ & $\begin{array}{r}\bar{r} \\
(\mathrm{pc})\end{array}$ & $\begin{array}{r}\bar{U} \\
\left(\mathrm{~km} \mathrm{~s}^{-1}\right)\end{array}$ & $\begin{array}{r}\bar{V} \\
\left(\mathrm{~km} \mathrm{~s}^{-1}\right)\end{array}$ & $\begin{array}{r}\bar{W} \\
\left(\mathrm{~km} \mathrm{~s}^{-1}\right)\end{array}$ & $\begin{array}{r}\text { Age } \\
(\mathrm{Myr})\end{array}$ & $N$ & $N_{\mathrm{r}}$ & $N_{\mathrm{k}}$ \\
\hline \multicolumn{2}{|c|}{ TW Hya } & $-21_{(22)}$ & $-53_{(23)}$ & $21_{(7)}$ & $63_{(30)}$ & $-9.7_{(4.1)}$ & $-17.1_{(3.1)}$ & $-4.8_{(3.7)}$ & 8 & 39 & 19 & 17 \\
\hline \multicolumn{2}{|c|}{ Tuc-Hor/GAYA } & $-12_{(22)}$ & $-24_{(11)}$ & $-34(8)$ & $49(8)$ & $-10.1_{(2.4)}$ & $-20.7_{(2.3)}$ & $-2.5_{(3.8)}$ & 20 & 52 & 50 & 44 \\
\hline \multicolumn{2}{|c|}{$\beta$ Pic-Cap } & $-9_{(27)}$ & $-5_{(14)}$ & $-15_{(10)}$ & $35_{(11)}$ & $-10.8_{(3.4)}$ & $-15.9(1.2)$ & $-9.8_{(2.5)}$ & 12 & 33 & 24 & 24 \\
\hline \multicolumn{2}{|c|}{$\epsilon$ Cha } & $-47(8)$ & $-80_{(14)}$ & $-25(5)$ & $96_{(17)}$ & $-8.6_{(3.6)}$ & $-18.6_{(0.8)}$ & $-9.3_{(1.7)}$ & 10 & 16 & 6 & 5 \\
\hline \multicolumn{2}{|c|}{$\eta$ Cha } & -33 ( 2) & $-80_{(5)}$ & $-34_{(2)}$ & $93(6)$ & $-12.2_{(0.0)}$ & $-18.1_{(0.9)}$ & $-10.1_{(0.5)}$ & 10 & 18 & 3 & 2 \\
\hline \multicolumn{2}{|c|}{ HD 141569} & -77 ( 3$)$ & $10(8)$ & $64(8)$ & $101_{(8)}$ & $-5.4_{(1.5)}$ & $-15.6_{(2.6)}$ & $-4.4_{(0.8)}$ & 5 & 5 & 3 & 2 \\
\hline \multicolumn{2}{|c|}{ Ext. R CrA } & $-97_{(44)}$ & $-1_{(4)}$ & $-30_{(16)}$ & $102_{(47)}$ & $-0.1_{(6.4)}$ & $-14.8_{(1.4)}$ & $-10.1_{(3.3)}$ & 13 & 59 & 7 & 4 \\
\hline \multicolumn{2}{|c|}{ AB Dor } & $6_{(21)}$ & $4_{(17)}$ & $-12_{(16)}$ & $32_{(13)}$ & $-7.4_{(3.2)}$ & $-27.4_{(3.2)}$ & $-12.9_{(6.4)}$ & $30-150$ & 40 & 36 & 35 \\
\hline \multirow[t]{3}{*}{ US } & Z99 & $-141_{(34)}$ & $-22_{(11)}$ & $50_{(16)}$ & $145_{(2)}$ & & & & $5-6$ & 120 & & \\
\hline & $\mathrm{M} 02^{1}$ & $-138_{(27)}$ & $-22_{(10)}$ & $49_{(12)}$ & $149_{(28)}$ & -0.9 & -16.9 & -5.2 & & 120 & & \\
\hline & S03 & & & & & $-6.7_{(5.9)}$ & $-16.0_{(3.5)}$ & $-8.0_{(2.7)}$ & $8-10$ & 155 & & \\
\hline \multirow[t]{3}{*}{$\mathrm{UCL}$} & Z99 & $-122_{(30)}$ & $-69_{(26)}$ & $32_{(16)}$ & $140_{(2)}$ & & & & $14-15$ & 221 & & \\
\hline & $\mathrm{M} 02^{1}$ & $-121_{(26)}$ & $-68_{(21)}$ & $32(15)$ & $145_{(24)}$ & -7.9 & -19.0 & -5.7 & & 218 & & \\
\hline & S03 & & & & & $-6.8_{(4.6)}$ & $-19.3_{(4.7)}$ & $-5.7_{(2.5)}$ & $16-20$ & 262 & & \\
\hline \multirow[t]{3}{*}{ LCC } & Z99 & $-62_{(18)}$ & $-102_{(24)}$ & $14_{(16)}$ & $118_{(2)}$ & & & & $11-12$ & 180 & & \\
\hline & $\mathrm{M} 2^{1}$ & $-61_{(14)}$ & $-100_{(15)}$ & $14_{(15)}$ & $120_{(18)}$ & -11.8 & -15.0 & -6.7 & & 179 & & \\
\hline & S03 & & & & & $-8.2_{(5.1)}$ & $-18.6_{(7.3)}$ & $-6.4_{(2.6)}$ & $16-20$ & 192 & & \\
\hline
\end{tabular}

${ }^{1}$ M02 derived an internal velocity dispersion among individual stars of $1.33 \mathrm{~km} \mathrm{~s}^{-1}$ for US, $1.23 \mathrm{~km} \mathrm{~s}^{-1}$ for UCL and $1.13 \mathrm{~km} \mathrm{~s}{ }^{-1}$ for LCC .

the stars belonging to this association with masses between 0.1 and $20 M_{\odot}$.

Z99, searching in the Hipparcos catalogue, found 120 members of US, 221 of UCL and 180 of LCC, with mean trigonometric distances (corrected for systematic effects) of $145 \pm 2$, $140 \pm 2$ and $118 \pm 2 \mathrm{pc}$, respectively (see Table 1). With regard to kinematics, all three associations have large velocity components in the direction away from the Sun, classically associated with the expansion motion of the Gould Belt (see for example Pöppel 1997; but also see Torra et al. 2000). Z99 provide mean proper motions from the Hipparcos catalogue and radial velocities from the Hipparcos Input catalogue for the stars identified as members of US, UCL and LCC. Madsen et al. (2002, hereafter M02) obtain astrometric radial velocities for the members of the Sco-Cen associations identified by Z99, assuming that all members share the same velocity vector. S03 compile radial velocities for the Sco-Cen members and derive from them the mean spatial motion, using Hipparcos parallaxes and proper motions. The mean spatial motions and standard deviations obtained by these authors are shown in Table 1 . We can see that the results are quite similar, except for the case of US, where there is a clear discrepancy in the $U$ value. As stated by M02, US is close to the limit of their method (larger distance, smaller angular size and smaller number of member stars, in comparison with LCC and UCL); thus, significant biases may be introduced into the solution they obtained for this association. Therefore, hereafter in this paper the Sco-Cen kinematics from S03 have been used to compute the back-tracing motion of the three associations. We have only used Madsen's data in Table 5.

\subsection{Young local associations}

A decade ago, very few PMS stars had been identified less than $100 \mathrm{pc}$ from the Sun. Nearly all the youngest stars $(\$ 30 \mathrm{Myr})$ studied then were located more than $140 \mathrm{pc}$ away in the molecular clouds of Taurus, Chamaeleon, Lupus, Sco-Cen and R CrA (all of them regions of recent star formation). The crosscorrelation of the Hipparcos and ROSAT catalogues suddenly changed this; a few stars were identified as very young (from their X-ray emission and lithium content) but closer than $100 \mathrm{pc}$, where there are no molecular clouds with SFR (see for example Neuhäuser \& Brandner 1998). Two explanations for the existence of these young stars far away from SFR were proposed. Sterzik \& Durisen (1995) suggested that the stars were formed in molecular clouds and later ejected as high-velocity stars during the decay of young multiple star systems. Feigelson (1996) suggested that the stars were formed inside small molecular clouds (or cloudlets), which later dispersed among the ISM and therefore can no longer be detected.

These young nearby stars were grouped into clusters, associations and moving groups, each with a few dozen members. Different approaches were used in each YLA discovery, but most of them made use of Hipparcos proper motions, X-ray emission (as a youth indicator), infrared emission (youth indicator) and ground-based spectroscopy ( $\mathrm{H} \alpha$ lines: youth indicator, $\mathrm{Li}$ lines: age estimation, radial velocities) and photometry (ages). We have compiled all the published YLA data in Appendix A. Table 1 shows the mean spatial and kinematic properties, ages and number of members for each YLA. The adopted age shown in Table 1 is that assigned for back-tracing the association orbits in the next section (see Table 2 for an exhaustive list of estimated values found in the literature). More information and tables containing all the data (in ASCII format) are available on a webpage we have published ${ }^{1}$.

Different nomenclature is used by the authors to define each YLA. The nature of the $\eta$ Cha cluster is clear: the stars are compacted in a region a very few pc in diameter. For the other groups, authors used the terms association, moving group or even system (for HD 141569, with only 5 known members). In this paper we have respected the denomination for each YLA used in the literature. However, the difference between the terms association and moving group applied to the different entities is not clear, since their spatial dispersions and kinematics are very similar (and if slight differences are apparent, they do not correspond to the classifications association or moving group; see Table 1).

Except for the case of AB Dor, all YLA studied here are younger than $\sim 30 \mathrm{Myr}$ (even considering the large uncertainties

1 http://www.am.ub.es/ dfernand/YLA 
Table 2. Estimated ages for the YLA published in the literature.

\begin{tabular}{|c|c|c|c|}
\hline Association & Estimated age & Method & Reference \\
\hline \multirow[t]{8}{*}{ TW Hya } & $10_{-5}^{+10} \mathrm{Myr}$ & Spectroscopy + HR diagram (BVI) & Soderblom et al. (1998) \\
\hline & $\sim 8 \mathrm{Myr}$ & HR diagram (JHK) & Webb et al. (1999) \\
\hline & $5-15 \mathrm{Myr}$ & HD diagram (IR) & Weintraub et al. (2000) \\
\hline & $8.3 \mathrm{Myr}$ & Expansion age & Makarov \& Fabricius (2001) \\
\hline & $4.7 \pm 0.6 \mathrm{Myr}$ & Expansion age & Makarov et al. (2005) \\
\hline & $20_{-7}^{+25} \mathrm{Myr}$ & Expansion age & Mamajek (2005) \\
\hline & $8.3^{-1} \pm 0.8 \mathrm{Myr}$ & Dynamical age & de la Reza et al. (2006) \\
\hline & $10_{-7}^{+10} \mathrm{Myr}$ & Spec. + HR diagram (VIJK) $+\mathrm{Li}+\mathrm{H} \alpha$ & Barrado y Navascués (2006) \\
\hline \multirow[t]{6}{*}{ Tuc-Hor/GAYA } & $\sim 40 \mathrm{Myr}$ & $\mathrm{H} \alpha$ emission & Zuckerman \& Webb (2000) \\
\hline & $10-30 \mathrm{Myr}$ & $\mathrm{X}$-ray emission & Stelzer \& Neühauser (2000) \\
\hline & $\sim 30 \mathrm{Myr}$ & Kinematic + HR diagram (BV) & Torres et al. (2000) \\
\hline & $10-40 \mathrm{Myr}$ & HR diagram (VRI) & Zuckerman et al. (2001b) \\
\hline & $\sim 20 \mathrm{Myr}$ & Kinematic age & Torres et al. (2001) \\
\hline & $27 \mathrm{Myr}$ & Dynamical age & Makarov (2007) \\
\hline \multirow[t]{5}{*}{$\beta$ Pic-Cap } & $20 \pm 10 \mathrm{Myr}$ & HR diagram (BVI) & Barrado y Navascués (1999) \\
\hline & $12_{-4}^{+8} \mathrm{Myr}$ & $\mathrm{HR}$ diagram $(\mathrm{VI})+\mathrm{Li}$ & Zuckerman et al. (2001a) \\
\hline & $11.2 \pm 0.3 \mathrm{Myr}$ & Dynamical age & Ortega et al. (2002) \\
\hline & $12 \mathrm{Myr}$ & Dynamical age & Song et al. (2003) \\
\hline & $22 \pm 12 \mathrm{Myr}$ & Dynamical age & Makarov (2007) \\
\hline \multirow[t]{3}{*}{$\epsilon$ Cha } & $5-15 \mathrm{Myr}$ & HR diagram (BV) & Terranegra et al. (1999) \\
\hline & $3-5 \mathrm{Myr}$ & HR diagram (VI) & Feigelson et al. (2003) \\
\hline & $7 \mathrm{Myr}$ & Dynamical age & Jilinski et al. (2005) \\
\hline \multirow[t]{5}{*}{$\eta$ Cha } & $2-18 \mathrm{Myr}$ & HR diagram $(\mathrm{R})+\mathrm{Li}$ & Mamajek et al. (1999) \\
\hline & $10-15 \mathrm{Myr}$ & Dynamical age & Mamajek et al. (2000) \\
\hline & $5 \pm 4 \mathrm{Myr}$ & HR diagram (VRI) & Lawson et al. (2001) \\
\hline & $6_{-1}^{+2} \mathrm{Myr}$ & HR diagram (JHK) & Luhman \& Steeghs (2004) \\
\hline & $7 \mathrm{Myr}$ & Dynamical age & Jilinski et al. (2005) \\
\hline \multirow[t]{2}{*}{ HD 141569} & $5 \pm 3 \mathrm{Myr}$ & X-ray + Li + HR diagram (JHK) & Weinberger et al. (2000) \\
\hline & $4.7 \pm 0.3 \mathrm{Myr}$ & Spectroscopy + Kurucz's (1993) models & Merín et al. (2004) \\
\hline \multirow[t]{2}{*}{ Ext. R CrA } & $10-15 \mathrm{Myr}$ & HR diagram (BVRI-JHK) & Neuhaüser et al. (2000) \\
\hline & $\sim 15 \mathrm{Myr}$ & Dynamical age & Quast et al. (2001) \\
\hline \multirow[t]{5}{*}{ AB Dor } & $\sim 50 \mathrm{Myr}$ & $\mathrm{H} \alpha$ emission & Zuckerman et al. (2004) \\
\hline & $75-150 \mathrm{Myr}$ & HR diagram (VK) & Luhman et al. (2005) \\
\hline & $30-50 \mathrm{Myr}$ and $80-120 \mathrm{Myr}$ (2 subgroups) & $\mathrm{HR}$ diagram $(\mathrm{VI})+\mathrm{Li}$ & López-Santiago et al. (2006) \\
\hline & $38 \mathrm{Myr}$ & Dynamical age & Makarov (2007) \\
\hline & $118 \pm 20 \mathrm{Myr}$ & Dynamical age & Ortega et al. (2007) \\
\hline
\end{tabular}

in the age estimations published in the literature; see Table 2). The Tuc-Hor/GAYA association has an estimated age of about 20 Myr, whereas the other YLA (TW Hya, $\beta$ Pic-Cap, $\epsilon$ Cha, $\eta$ Cha, HD 141569 and Ext. R CrA) are younger than $\sim 15 \mathrm{Myr}$ and of similar ages to (or younger than) the Sco-Cen associations. (The AB Dor moving group is clearly older and will not be used in our analysis; see, however, López-Santiago et al. 2006; and Makarov 2007.)

At present, all YLA members in our compendium are spread over a region of about $120 \times 130 \times 140 \mathrm{pc}$, mainly concentrated in the fourth Galactic quadrant, and mostly in the southern celestial hemisphere (see Fig. 2). The spatial distributions within each association reach a few tens of pc in the Galactic plane, and less than $20 \mathrm{pc}$ in the perpendicular direction (see Table 1).

The heliocentric velocity components of all the associations are very similar, $(U, V, W) \sim[-(9-12),-(16-21),-(3-$ 10)] $\mathrm{km} \mathrm{s}^{-1}$ (except for the extended R CrA association and, maybe, the HD 141569 system). To obtain these mean values we used all the stars for which complete kinematic data are available. We checked that rejecting those stars with the larger residuals does not modify the mean values by more than $1.0 \mathrm{~km} \mathrm{~s}^{-1}$, though the standard deviations are clearly reduced (in most cases less than $2.0 \mathrm{~km} \mathrm{~s}^{-1}$ for all the components). The only exception is the extended $\mathrm{R} \mathrm{CrA}$ association. It contains only 5 stars with complete kinematic data and there are large uncertainties in their radial velocities. This results in large standard deviations in the velocity components, especially in $U$, and prevents us from using this association in our analysis.

The resulting YLA velocity components are very similar to those of the associations in the Sco-Cen complex (see Table 1). Taking into account the similar kinematics observed among YLA, and the observation that most of them are nearly coeval (ages of $\sim 5-15 \mathrm{Myr}$ ), it is interesting to consider whether we can really speak of distinct entities. Figure 3 shows the velocity distribution of YLA member stars for which complete kinematic data are available. Using a kernel estimation, several of the associations are clearly distinguished kinematically: we can clearly identify peaks corresponding to Tuc-Hor/GAYA, $\beta$ Pic-Cap, $\epsilon$ Cha and $\eta$ Cha. The HD 141569 system is not identified in Fig. 3 due to the small number of members whose complete kinematic data are available (only 2 stars).

Initially, it is surprising that the TW Hya association is not evident in Fig. 3, and even a local minimum in density can be observed near its expected positions $([U, V, W] \sim$ $[-10,-17,-5] \mathrm{km} \mathrm{s}^{-1}$; see Table 1$)$. This may be due to a combination of three factors: its position in the $(U V W)$ planes very near other YLA, its relatively high total velocity dispersion $\sigma$ (computed as $\sigma=\sqrt{\sigma_{U}^{2}+\sigma_{V}^{2}+\sigma_{W}^{2}}$ and compared with its neighbours in these planes), and the relatively low number of TW Hya stars with complete kinematic data $\left(N_{\mathrm{k}}\right.$ in Table 1$)$ 

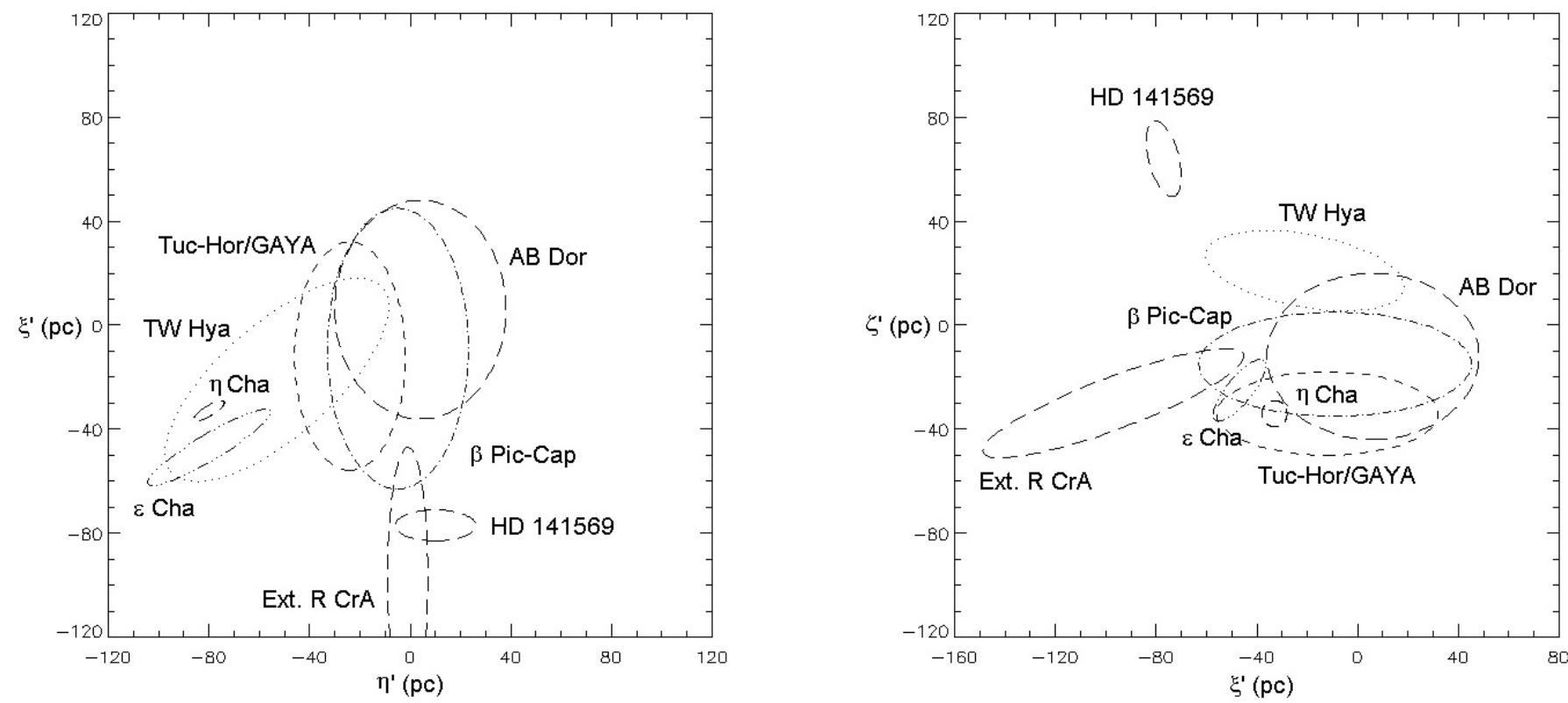

Fig. 2. Locations of the young local associations projected onto the Galactic (left) and meridian (right) planes. The size of the ellipses represents the projected dimension of the associations (more than $90 \%$ of the stars inside the plotted area). $\xi^{\prime}$ is pointing to the Galactic anti-centre, $\eta^{\prime}$ is the direction of Galactic rotation and $\zeta^{\prime}$ is pointing to the north Galactic pole.
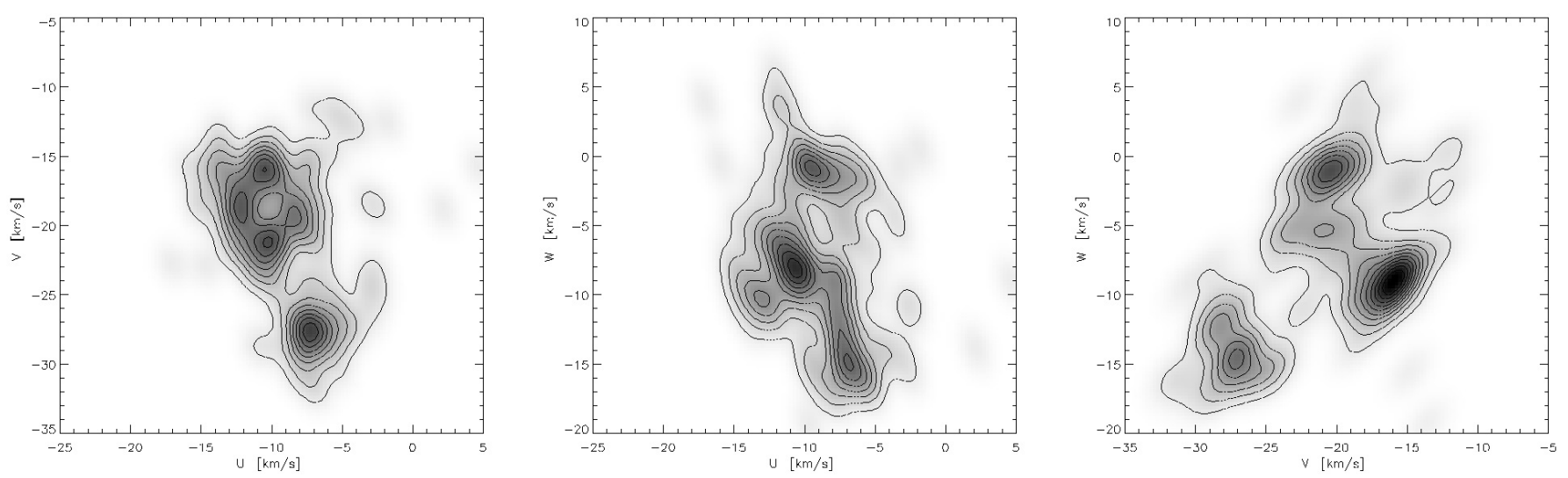

Fig. 3. Distribution in the $(U-V)[l e f t],(U-W)[$ middle $]$ and $(V-W)[$ right $]$ planes of heliocentric velocity components for YLA member stars whose complete kinematic data are available. A kernel estimator was used to indicate the isocontours.

compared to Tuc-Hor/GAYA and $\beta$ Pic-Cap associations (resulting in a low contribution to the probability density function shown in Fig. 3).

To study this case in more detail, Fig. 4 shows the velocity distribution $[(U, V)$ and $(U, W)$ planes] of the stars belonging to the three associations with the most complete kinematic data: TW Hya, Tuc-Hor/GAYA and $\beta$ Pic-Cap. At first sight it is clear that the two entities Tuc-Hor/GAYA and $\beta$ Pic-Cap are much better defined in these planes than the TW Hya association is. The main kernel estimator peaks agree with the mean values shown in Table $1,(\bar{U}, \bar{V}, \bar{W})$ for these two associations. However, in the case of TW Hya, no central peak is found at the expected position on the planes for the mean values. In Fig. 5 we show the positions in the $(U, V)$ plane for the stars belonging to the TW Hya association, and we compare them with the mean velocity components for the YLA. Whereas the other YLA have their own place in this plane (with low superposition of their error bars), the TW Hya association seems to fill a region shared by other YLA. We have computed the distance in the $(U V W)$ velocity space from each TW Hya star to the position of the mean velocity of the YLA. Only 4 out of 17 stars are nearer to the position of the TW Hya association in the $(U V W)$ space than to other YLA. In Table 3 we have done the exercise of recomputing the mean velocity components and their dispersions for the YLA after adding the TW Hya stars to the nearest YLA in the $(U V W)$ space. We can see that the change in the mean velocity vectors of the associations is much smaller than their corresponding standard deviation. A controversial hypothesis one could propose is that the TW Hya association (the first one discovered among the YLA) could not actually be an association at all, and one may even wonder if its member stars could be redistributed among the other YLA. However, there are two important facts that do not support this hypothesis. The first one concerns the spatial distribution of the YLA at present. As can be seen in Fig. 2, TW Hya is clearly isolated in the $(X, Z)$ plane (not considering the much older members of the AB Dor moving group), with a spatial extent in agreement with that observed in the other YLA. The previously discussed kinematic reassigment 

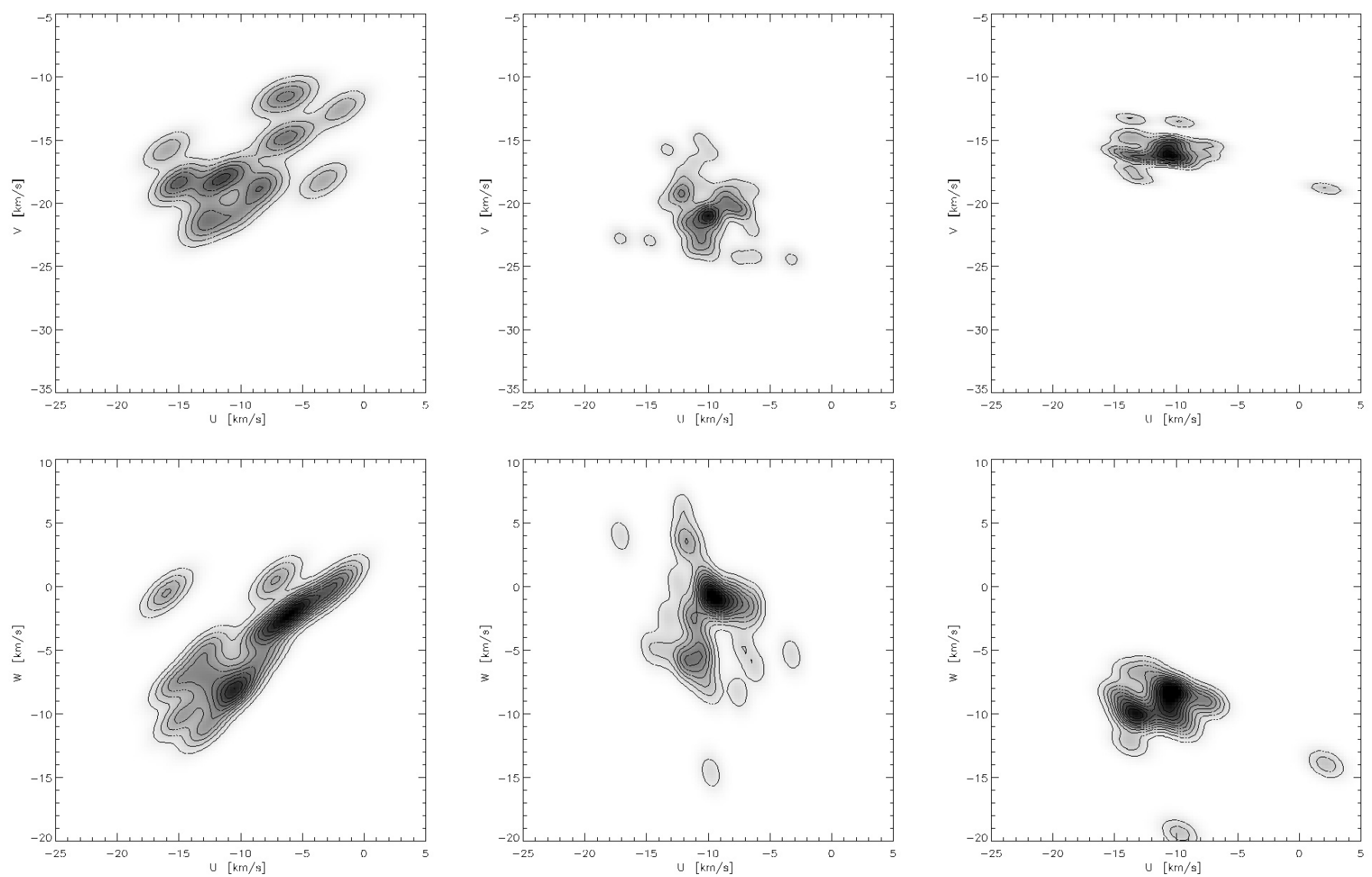

Fig. 4. Distribution in the $(U, V)[$ top row $]$ and $(U, W)$ [bottom row $]$ planes of heliocentric velocity components for those stars with complete kinematic data belonging to the TW Hya association (left column), the Tuc-Hor/GAYA association (middle column) and the $\beta$ Pic-Cap moving group (right column).

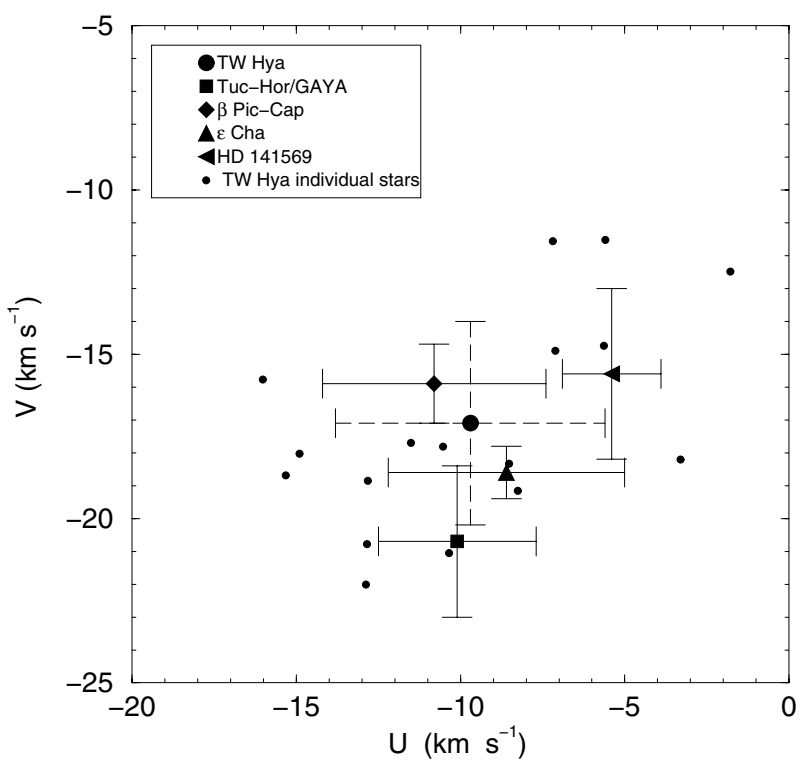

Fig. 5. Mean helicocentric velocity components of the YLA (indicating their $1 \sigma$ error bars; standard deviation) and position in the $(U, V)$ plane of those stars (with complete kinematic data) belonging to the TW Hya association.

of stars would enlarge, clearly in excess, the present accepted size for the other associations. The second important fact is that, whereas almost all Tuc-Hor/GAYA and $\beta$ Pic-Cap members have Hipparcos parallaxes, $70 \%$ of the TW Hya members have only an estimated distance from broadband photometry (Song et al. 2003). The assumption of a relative error of about $20 \%$ for this parameter would have a contribution of up to $3-4 \mathrm{~km} \mathrm{~s}^{-1}$ in the velocity dispersion components. This value, convolved with an intrisic velocity dispersion of about $1 \mathrm{~km} \mathrm{~s}^{-1}$ (Mamajek 2005), would justify the values shown in Table 1 . Furthermore, we have confirmed that the discrepancies between these photometric distances and the moving cluster distances by Mamajek (2005) are about $20 \%$. From the above arguments it is clear that more accurate astrometric data is mandatory to clarify both the kinematic dispersion and membership assignment of TW Hya members. The large dispersion observed in the estimated ages for this association (see Table 2) also contributes to the deficient characterisation of this entity.

\section{Stellar orbits}

The integration back in time of the Sco-Cen and YLA orbits allows us to study their origin and possible influence on the immediate ISM over the last million years. To compute the stellar orbits back in time we used the code developed by Asiain et al. (1999b) based on the integration of the equations of motion using a realistic model of the Galactic gravitational potential.

If we consider a coordinate system $(\xi, \eta, \zeta)^{2}$ centred on the Sun and rotating around Galactic centre with a constant angular

\footnotetext{
${ }^{2} \xi$ points towards Galactic centre, $\eta$ in the direction of Galactic rotation and $\zeta$ towards the Galactic north pole.
} 
Table 3. Mean heliocentric velocity components (and their standard deviations) of the young local associations before and after adding those stars belonging to TW Hya (see text).

\begin{tabular}{lcccc}
\hline \hline Association & $\begin{array}{r}\bar{U} \\
\left(\mathrm{~km} \mathrm{~s}^{-1}\right)\end{array}$ & $\begin{array}{r}\bar{V} \\
\left(\mathrm{~km} \mathrm{~s}^{-1}\right)\end{array}$ & $\begin{array}{r}\bar{W} \\
\left(\mathrm{~km} \mathrm{~s}^{-1}\right)\end{array}$ & $N_{\mathrm{k}}$ \\
\hline Tuc-Hor/GAYA & $-10.1_{(2.4)}$ & $-20.7_{(2.3)}$ & $-2.5_{(3.8)}$ & 44 \\
adding stars & $-10.2_{(2.6)}$ & $-20.5_{(2.3)}$ & $-2.5_{(3.7)}$ & 47 \\
\hline$\beta$ Pic-Cap & $-10.8_{(3.4)}$ & $-15.9_{(1.2)}$ & $-9.8_{(2.5)}$ & 24 \\
adding stars & $-11.2_{(3.3)}$ & $-16.3_{(1.4)}$ & $-9.2_{(1.6)}$ & 28 \\
\hline$\epsilon$ Cha & $-8.6_{(3.6)}$ & $-18.6_{(0.8)}$ & $-9.3_{(1.7)}$ & 5 \\
adding stars & $-9.5_{(3.0)}$ & $-19.2_{(1.4)}$ & $-8.9_{(1.9)}$ & 9 \\
\hline HD 141569 & $-5.4_{(1.5)}$ & $-15.6_{(2.6)}$ & $-4.4_{(0.8)}$ & 2 \\
adding stars & $-5.2_{(1.9)}$ & $-14.3_{(2.5)}$ & $-1.9_{(1.9)}$ & 8 \\
\hline
\end{tabular}

Table 4. Position and velocity of LSR, and local density (Kerr \& Lynden-Bell 1986), bulge, disk and halo parameters (Miyamoto \& Nagai 1975; Allen \& Santillán 1991), together with spiral structure (Fernández et al. 2001) and central bar (Binney et al. 1991; Palouš et al. 1993) parameters used in the integration of stellar orbits.

\begin{tabular}{ll}
\hline \hline$R_{\odot}$ & $8.5 \mathrm{kpc}$ \\
$\Theta_{\odot}$ & $220 \mathrm{~km} \mathrm{~s}^{-1}$ \\
$\rho_{\odot}$ & $0.15 M_{\odot} \mathrm{pc}^{-3}$ \\
\hline$M_{\mathrm{B}}$ & $1.4 \times 10^{10} M_{\odot}$ \\
$M_{\mathrm{D}}$ & $8.6 \times 10^{10} M_{\odot}$ \\
$M_{\mathrm{H}}$ & $1.1 \times 10^{11} M_{\odot}$ \\
$a_{\mathrm{B}}$ & $0.39 \mathrm{kpc}$ \\
$a_{\mathrm{D}}$ & $5.3 \mathrm{kpc}$ \\
$b_{\mathrm{D}}$ & $0.25 \mathrm{kpc}$ \\
$a_{\mathrm{H}}$ & $12.0 \mathrm{kpc}$ \\
\hline$m$ & 2 \\
$i$ & $-6^{\circ}$ \\
$\psi_{\odot}$ & $330^{\circ}$ \\
$f_{\mathrm{r}}$ & 0.05 \\
$\Omega_{\mathrm{p}}$ & $30 \mathrm{~km} \mathrm{~s}^{-1} \mathrm{kpc}^{-1}$ \\
\hline$a_{\mathrm{B}} / b_{\mathrm{B}}$ & 2.381 \\
$a_{\mathrm{B}} / c_{\mathrm{B}}$ & 3.030 \\
$q_{\mathrm{B}}$ & $5 \mathrm{kpc}$ \\
$t_{o}$ & $5 \times 10^{8} \mathrm{years}^{\circ}$ \\
$\theta_{o}$ & $45^{\circ}$ \\
$\Omega_{\mathrm{B}}$ & $70 \mathrm{~km} \mathrm{~s}^{-1} \mathrm{kpc}^{-1}$ \\
\hline
\end{tabular}

velocity $\Omega_{\odot}$, the equations of motion of a star are:

$\ddot{\xi}=-\frac{\partial \Phi}{\partial \xi}-\Omega_{\odot}^{2}\left(R_{\odot}-\xi\right)-2 \Omega_{\odot} \dot{\eta}$

$\ddot{\eta}=-\frac{\partial \Phi}{\partial \eta}+\Omega_{\odot}^{2} \eta+2 \Omega_{\odot} \dot{\xi}$

$\ddot{\zeta}=-\frac{\partial \Phi}{\partial \zeta}$

where $\Phi=\Phi(R, \theta, z ; t)$ is the gravitational potential of the Galaxy in galactocentric cylindrical coordinates.

When $\Phi$ is a known function, these equations can be solved numerically through a fourth-order Runge-Kutta integrator. We have decomposed $\Phi$ into three components: the general axisymmetric potential $\Phi_{\mathrm{AS}}$ (Allen \& Santillán 1991), the potential due to the spiral structure of the Galaxy $\Phi_{\mathrm{Sp}}$, and that due to the central bar $\Phi_{\mathrm{B}}$ (see Table 4). In this way we get a realistic estimate of the Galactic gravitational potential.

The spiral structure potential is taken from Lin's theory (Lin \& Shu 1964; Lin et al. 1969):

$\Phi_{\mathrm{Sp}}(\mathrm{R}, \theta ; t)=\mathcal{A} \cos \left[m\left(\Omega_{p} t-\theta\right)+\psi(R)\right]$

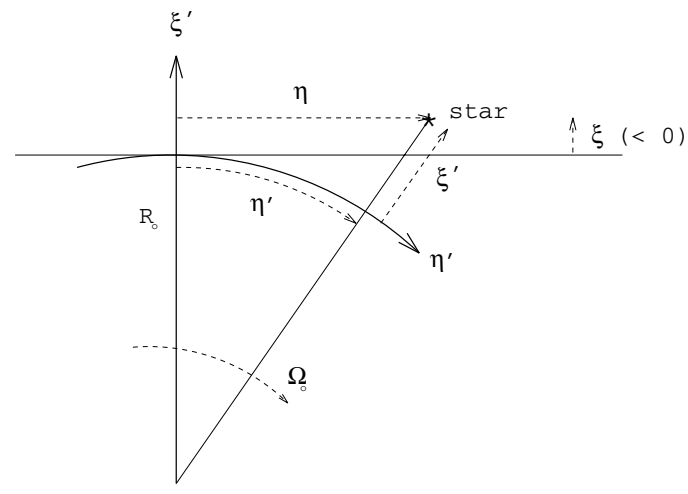

Fig. 6. Heliocentric coordinates $(\xi, \eta, \zeta)$ and $\left(\xi^{\prime}, \eta^{\prime}, \zeta^{\prime}\right)$ (from Asiain 1998; see also Asiain et al. 1999b).

where:

$$
\begin{aligned}
\mathcal{A} & =\frac{\left(R_{\odot} \Omega_{\odot}\right)^{2} f_{\mathrm{r} 0} \tan i}{m} \\
\psi(R) & =-\frac{m}{\tan i} \ln \left(\frac{R}{R_{\odot}}\right)+\psi_{\odot}
\end{aligned}
$$

where $\mathcal{A}$ is the amplitude of the spiral potential, $f_{\mathrm{r} 0}$ the ratio between the radial component of the force due to the spiral arms and that due to the general Galactic field, $\Omega_{\mathrm{p}}$ the constant angular velocity of the spiral pattern, $m$ the number of spiral arms, $i$ the pitch angle of the arms, $\psi$ the radial phase of the spiral wave and $\psi_{\odot}$ its value at the position of the Sun. We have taken the values obtained by Fernández et al. (2001; see also Fernández 2005) for a model with 2 spiral arms.

In the case of the potential due to the central bar, we have chosen the triaxial ellipsoid model of Palous et al. (1993) with a rotation speed $\Omega_{\mathrm{B}}=70 \mathrm{~km} \mathrm{~s}^{-1} \mathrm{kpc}^{-1}$ (Binney et al. 1991). Although large uncertainties are still present in the bar parameters, this structure has an almost negligible effect on our stellar trajectories (as far back as $30 \mathrm{Myr}$; see Asiain 1998).

All the figures in the next section use the $\left(\xi^{\prime}, \eta^{\prime}, \zeta^{\prime}\right)$ coordinate system centred on the Sun's current position and rotating around Galactic centre at a constant velocity $\Omega_{\odot}$ (see Fig. 6). $\xi^{\prime}$ points to the Galactic anti-centre and is defined as $\xi^{\prime}=R-R_{\odot}$. $\eta^{\prime}$ is a linear coordinate, measured along the circle of radius $R_{\odot}$, which is positive in the direction of Galactic rotation. The coordinate system $\left(\xi^{\prime}, \eta^{\prime}, \zeta^{\prime}\right)$ is convenient for our purposes as it minimises variation in the variables.

We have integrated the orbits of the associations as a whole, using the mean position and velocity for each association shown in Table 1. The results are presented in Figs. 7 and 8, where not only do we consider the orbits in the Galactic plane (as is usual in the literature), but also in the meridian and rotation planes. In Fig. 7 we show the estimated error in the position of the associations at birth (grey area). These error areas have been obtained computing the orbits back in time, using the values $\bar{U} \pm 2 S e_{\bar{U}}$, $\bar{V} \pm 2 S e_{\bar{V}}$ as present velocity components (where $S e_{\bar{U}}$ and $S e_{\bar{V}}$ are the standard errors in the $\bar{U}, \bar{V}$ mean velocity components obtained from the standard deviations in Table 1). We have confirmed that uncertainties in the $\bar{W}$ component have a negligible effect on the orbits shown in Fig. $7^{3}$. An error in age shall be

\footnotetext{
${ }^{3}$ A variation of $\pm 2 S e_{\bar{W}}$ in $\bar{W}$ implies a change in the vertical position $\left(\zeta^{\prime}\right)$ with time, thus slightly affecting the radial and azimuthal forces acting on the star (see Eq. (9) in Asiain et al. 1999b; and Eqs. (1), (3) and (5) in Allen \& Santillán 1991). However, we have confirmed that this effect results in a change in position of less than $10^{-3} \mathrm{pc}$ in the $\left(\xi^{\prime}, \eta^{\prime}\right)$ plane in the present case.
} 


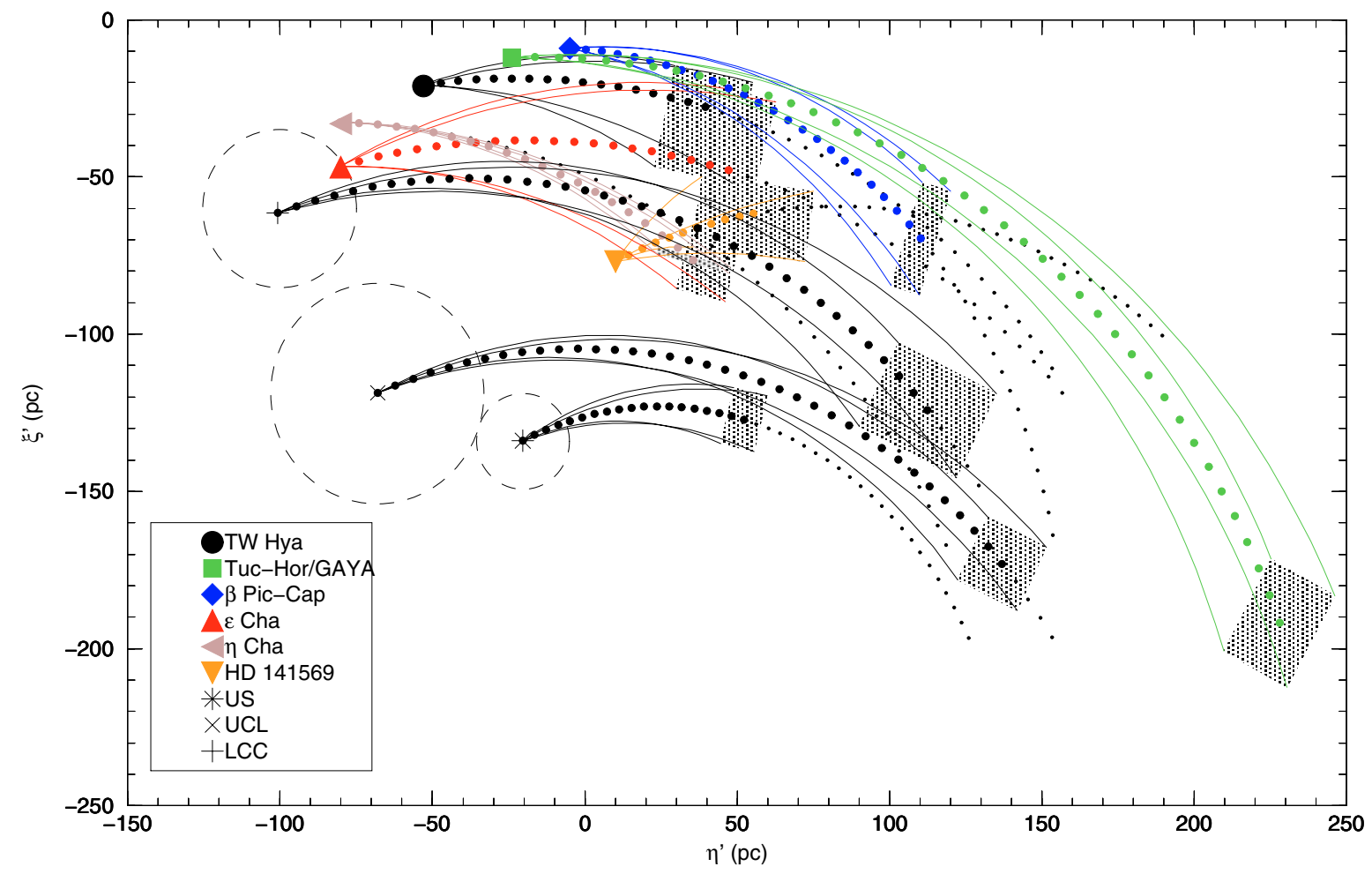

Fig. 7. Positions and orbits in the Galactic plane $\left(\xi^{\prime}, \eta^{\prime}\right)$ of YLA and the Sco-Cen complex going back in time to their individual ages. The orbits are also shown (small dots) as far back as -20 Myr. The grey areas show the expected positional errors at birth due to kinematic observational errors. The centre of the $\left(\xi^{\prime}, \eta^{\prime}\right)$ coordinate system is comoving with the LSR.

$\xi^{\prime}(\mathrm{pc})$

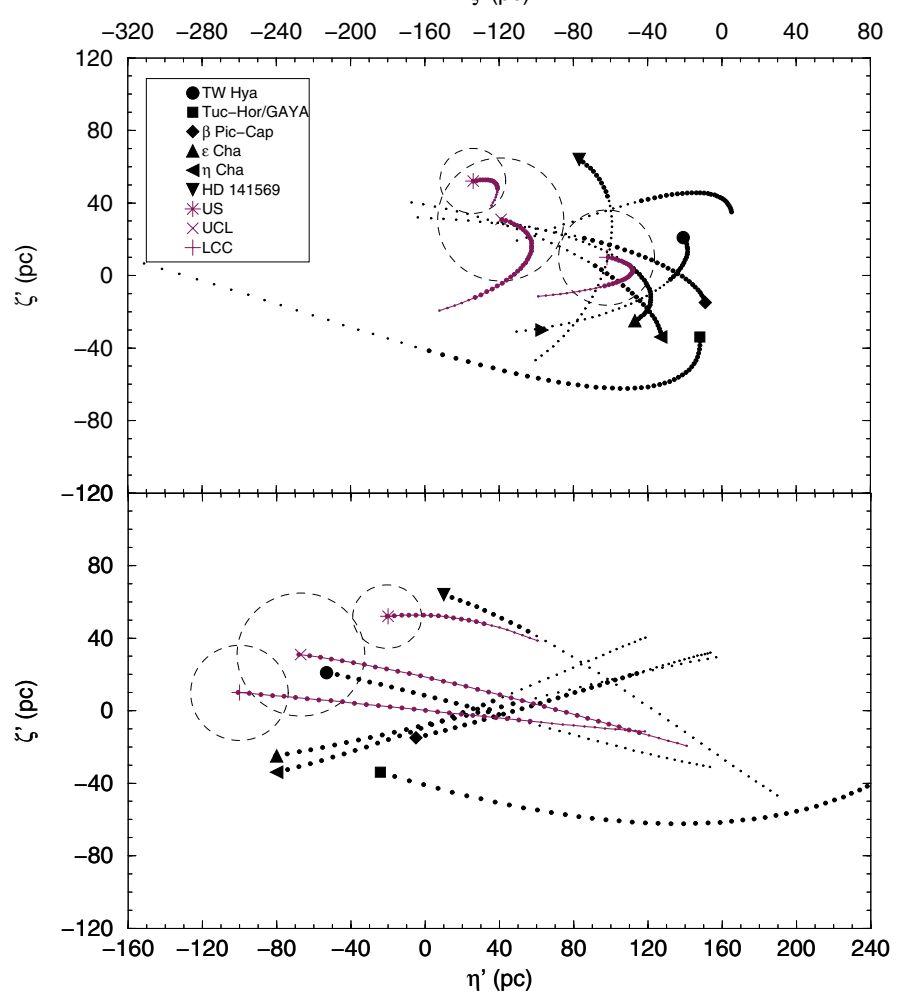

Fig. 8. Present positions and orbits in the Galactic planes $\left(\xi^{\prime}, \zeta^{\prime}\right)($ top $)$ and $\left(\eta^{\prime}, \zeta^{\prime}\right)($ bottom $)$ for YLA and the Sco-Cen complex. The error areas (not shown) are of similar size as in Fig. 7. (See comments at Fig. 7.)

read as a displacement of the error areas along the plotted orbits in the figure. Unfortunately, age uncertainties are large for all the associations (see Table 2). Later in this paper we study how these large uncertainties in age may affect our results.

The most obvious trend observed in Figs. 7 and 8 is the spatial concentration of all the associations (Sco-Cen complex and YLA) in the first Galactic quadrant in the past. All the YLA (except the relatively old Tuc-Hor/GAYA) were in the region of the Galactic plane bounded by $-70 \lesssim \xi^{\prime} \lesssim-30 \mathrm{pc}$ and $35 \lessgtr \eta^{\prime} \lesssim 110 \mathrm{pc}$ at their time of birth. There is a very conspicuous spatial grouping at the time of birth of TW Hya, $\epsilon$ Cha, $\eta$ Cha and HD 141569 (in a sphere 25 pc in radius). At its birth, $\beta$ Pic-Cap was located about $50 \mathrm{pc}$ from the other YLA. All the associations were concentrated in the region $0 \lesssim \zeta^{\prime} \lesssim 45 \mathrm{pc}$ at their birth. Therefore the associations were formed slightly above the Galactic plane (the Sun, whose position is the origin of the $\zeta^{\prime}$ coordinate, is located about 16 pc above the Galactic plane; see Binney \& Merrifield 1998). The region where these associations were formed had a size of $40 \times 75 \times 45 \mathrm{pc}\left(\xi^{\prime} \times \eta^{\prime} \times \zeta^{\prime}\right)$. At present, the stars belonging to YLA are distributed in a region of about $120 \times 130 \times 140 \mathrm{pc}$. The volume they occupy has therefore increased by a factor of $\sim 16$ since their birth. As can be observed in Fig. 7, the errors associated with the mean velocity components of the associations do not have a crucial influence on the previous results: the error areas have typical side lengths of about $10-30 \mathrm{pc}$.

In the case of the Sco-Cen associations, both UCL and LCC began life very near the Galactic plane; for both of them $-19 \lesssim$ $\zeta^{\prime} \lesssim-12$ pc at birth (they were closer than $\sim 4$ pc to the Galactic plane). For US, $\zeta^{\prime} \sim 40 \mathrm{pc}$ at birth.

\section{Origin and evolution of the local structures}

The results obtained in the previous section suggest that the formation of YLA was triggered in a neighbouring region of the 
first Galactic quadrant between 5 and 15 Myr ago. In this section we present a description of this mechanism and link it to the origin and evolution of the Sco-Cen complex and the LB.

\subsection{Origin of the Sco-Cen complex}

The gas associated with the Sco-Cen complex has classically been considered as a part of the Lindblad Ring; a toroidal structure of HI and molecular clouds in expansion (Lindblad 1967) belonging to the Gould Belt. Close to Sco-Cen, the clouds of Lupus and $\rho$ Oph, and the so-called Aquila Rift, would also form part of the Lindblad Ring.

The structure that results from considering all these molecular complexes has a length of about $120^{\circ}$, and extends from the Vela region to the Aquila Rift. Below, we discuss the most plausible scenarios for star formation in this region and we conclude that the most likely is the collision of a GMC with a spiral arm. We mention the review published by S03, where other less plausible scenarios can be found.

\subsubsection{The sequential star-formation model}

This model was formulated by Blaauw $(1964,1991)$ and suggests that star formation begins at one end of a GMC and propagates to adjacent regions due to stellar winds and supernovae.

Preibisch \& Zinnecker (1999) later proposed a history of star formation in the Sco-Cen complex, using the classical ages of de Geus et al. (1989). Star formation in Sco-Cen would have begun around 15 Myr ago in UCL. 12 Myr ago, when star formation started in LCC, the most massive star in UCL would have exploded as a supernova, forming the largest HI shell surrounding the association. $5 \mathrm{Myr}$ ago, the shock front from this supernova would have passed through the cloud, which was to become the parent of US, triggering star formation there. Shortly after, the strong stellar winds from the most massive stars in US would begin to sweep away the molecular cloud, stopping the star formation process. Only 1.5 Myr ago, the most massive star in US would have exploded as a supernova, completely dispersing the US cloud. Nowadays, the wave front of this supernova would be travelling through the $\rho$ Oph molecular cloud, triggering star formation there.

Following S03, this model would result in systematic velocity fields with radial components directed away from the different centres of star formation. This is not what we observe in the results obtained in previous sections. However, the problem could be a little more complicated since the velocities of the young stars would not only depend on the velocity and direction of the shock front that compressed the parent cloud; the kinematics of the new stars would also depend on the original velocity of the molecular cloud, which could be similar to, or even larger than, the velocity of the shock front. Following this, the successive sources of star formation would not necessarily form groups of stars with clearly identified radial velocity components. Thus, contrary to the opinion of S03, we think that the sequential star-formation mechanism could have a crucial role in the global scenario for star formation in this region.

\subsubsection{The Gould Belt model based on an expanding gas ring}

The kinematics of the stars belonging to the Gould Belt seems compatible with the expansion model proposed by Olano (1982): a gas ring with a centre located at a distance of about $166 \mathrm{pc}$ in the direction $l \sim 131^{\circ}$. However, the Sco-Cen complex is precisely one of the regions of the Gould Belt that does not fit this model, since the observed proper motions are not orientated as predicted by Olano (see also Moreno et al. 1999). Moreover, as shown above, as we project the orbits of the stars in this region back in time, they no longer indicate the centre of the Gould Belt. S03 also mentions other problems, such as the fact that the age of the stars in Sco-Cen is an important fraction of the age of the Gould Belt predicted by this model (30 Myr), in spite of the large distance between the present position of Sco-Cen and the expansion centre of the Belt.

Furthermore, as shown in a previous paper (Torra et al. 2000), the Sco-Cen associations are not needed to explain the peculiar kinematics of the Gould Belt.

\subsubsection{Star formation triggered by a spiral arm shock wave}

The region in question could also have been formed as a result of the interaction of the parent GMC with the shock wave of a spiral arm. In this case, the main problem to be faced is that the classical view of the spiral structure of the Milky Way places the Sun in an interarm region, at about $1 \mathrm{kpc}$ from the nearest arm (see, for example, Georgelin \& Georgelin 1976) where there is no spiral shock wave capable of triggering star formation. However, in recent years there has been some evidence that this classical view could be wrong (see, for instance, Fernández et al. 2001, for a stellar kinematics study; or Amôres \& Lépine 2005 , for a model of interstellar extinction in the Galaxy), with the Sun closer to the inner (Sagittarius-Carina) arm. This forces us to study this possible scenario in more detail.

Figure 9 shows the orbits of YLA and the OB associations in Sco-Cen for times $0>t>-30 \mathrm{Myr}$ in a galactocentric reference frame $\left(X^{\prime}, Y^{\prime}\right)$ which is rotating with an angular velocity $\Omega=$ $\Omega_{\mathrm{p}}$ (the rotation velocity of the spiral arms of the Galaxy; so, in this system the spiral arms remain fixed). We used the value $\Omega_{\mathrm{p}}=30 \mathrm{~km} \mathrm{~s}^{-1} \mathrm{kpc}^{-1}$ obtained by Fernández et al. (2001). The Figure shows that, back in time, the orbits tend to concentrate these associations in a region with a phase of the spiral structure of $0^{\circ} \lesssim \psi \lesssim 10^{\circ}$, very near the spiral potential minimum at $\psi=$ $0^{\circ}$.

Following the non-linear density wave theory, the motion of the spiral structure in the Galactic plane generates a shock wave just before the arm potential minimum for regions outside the corotation radius (Roberts 1969; Bertin \& Lin 1996). In the case of our galaxy, the phase separation between the position of the shock wave and the potential minimum is not exactly known (see, for example, Gittins \& Clarke 2004). To quantify this, it is common to define the offset function $\Theta(R)=m\left(\theta_{\text {shock }}-\theta_{\text {min }}\right)$, where $m$ is the number of spiral arms of the Galaxy, and $\theta_{\text {shock }}$ and $\theta_{\min }$ are the Galactic longitude of the shock wave and the spiral potential minimum respectively, at galactocentric distance $R$. Roberts (1969) derives a small value for this function, close to 0. However, Shu et al. (1972) obtain $\Theta(R)=-72^{\circ}$, whereas Yuan $\&$ Grosbøl (1981) adopt $\Theta(R)=-30^{\circ}$. Despite there being no good agreement on the value of this parameter, it should have an absolute value of no more than a few tens of degrees.

Recent works have studied the formation of a GMC due to the arrival of the spiral shock wave, either gathering together preexisting molecular gas or inducing high densities in the shock, converting HI into HII (see Dobbs \& Bonnell 2007, and references herein). According to these authors, GMC formation occurs within a very few Myr ( $\$ 5 \mathrm{Myr})$ and star formation begins very quickly, $\sim 5 \mathrm{Myr}$ after the cloud formation (Clark et al. 2005). The first supernovae event occurs about 4 Myr after the formation of the very high mass stars, halting the star formation 

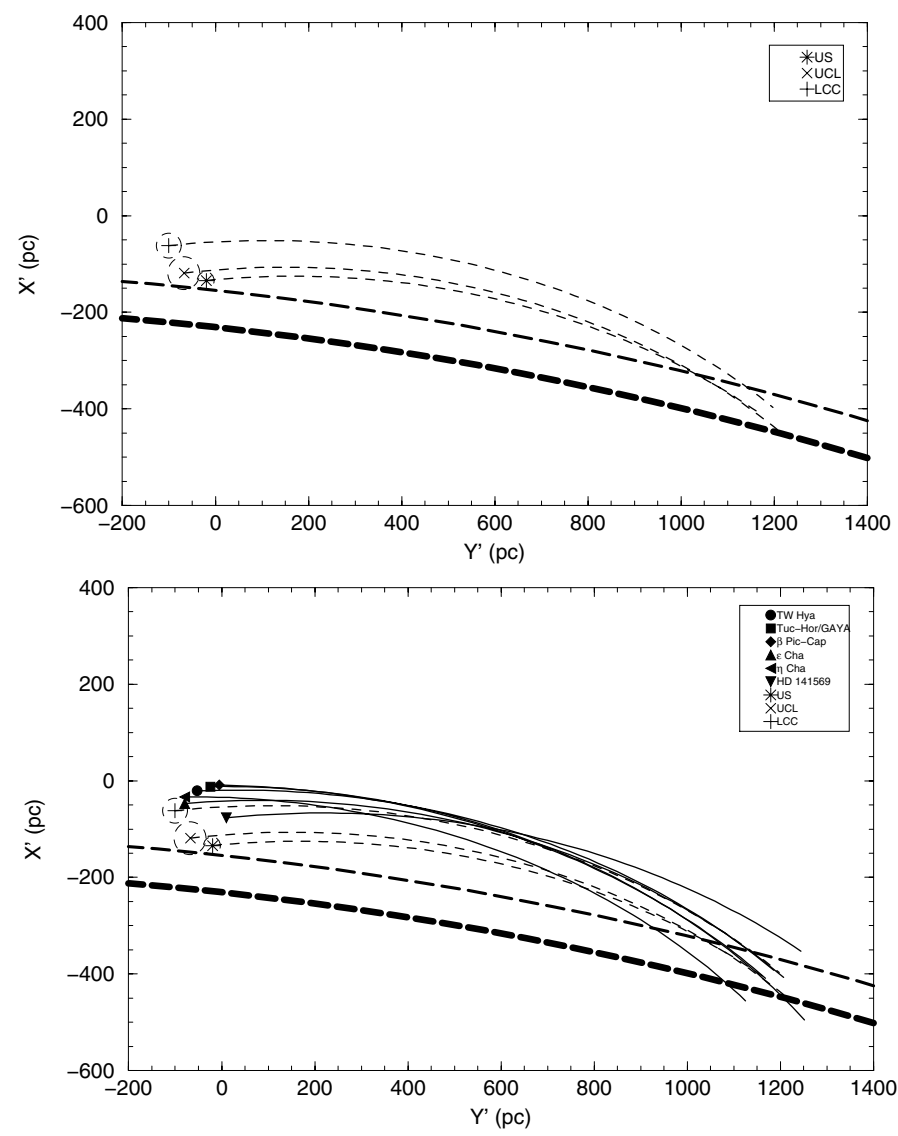

Fig. 9. Orbits in the Galactic plane $\left(X^{\prime}-Y^{\prime}\right)$ integrated back in time to $t=$ $-30 \mathrm{Myr}$ for YLA and the three associations of the Sco-Cen complex (below, only the orbits for Sco-Cen). The thick-dashed line shows the position of the minimum of the spiral potential (Fernández et al. 2001). The thin-dashed line is the position of the phase of the spiral structure $\psi=10^{\circ}$.

process. The stars of the OB association complex are then born, about $\sim 10-15$ Myr after the arrival of the spiral shock wave.

When the shock wave hits the gas, its direction of motion changes. Assuming that the shock is strong and sufficiently dissipative, the velocity component perpendicular to the spiral arm is strongly reduced (see for example Landau \& Lifshitz 1982). After interaction with the shock wave, the gas moves in a direction practically tangential to the spiral arm (see Fig. 10). Considering $\Omega_{\mathrm{p}}=30 \mathrm{~km} \mathrm{~s}^{-1} \mathrm{kpc}^{-1}$, in the vicinity of the Sun the shock wave is moving around $4 \mathrm{~km} \mathrm{~s}^{-1} \mathrm{kpc}^{-1}$ faster than the local standard of rest (LSR; equivalent to $30-35 \mathrm{~km} \mathrm{~s}^{-1}$, depending on the value adopted for the distance to Galactic centre) in the Galactic rotation direction. In this framework, if the shock wave of a spiral arm hits a molecular cloud, it is compressed, and star formation can be triggered.

This is the process proposed by S03 for the formation of the Sco-Cen complex. They assume that the Sun is placed in a region slightly inside the corotation radius. The gas and stars that follow the Galactic rotational motion are then moving faster than the shock wave. In this framework, with respect to a reference frame that is rotating at the same velocity as the spiral arms, the gas is slowed when it hits the shock wave, and afterwards it moves practically parallel to the spiral arm, approaching the Galactic centre (see Fig. 10, top). However, with respect to LSR, the compressed gas and the newly born stars have velocities in the direction opposite to the Galactic rotation and away from

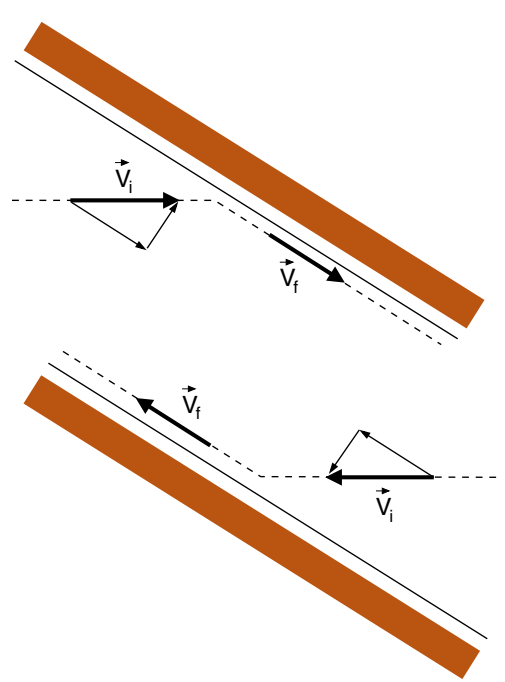

Fig. 10. Variation of the velocity vector of a test particle when it hits the shock wave (solid line) in the vicinity of the centre of a spiral arm (shadow) and in a region inside (top) and outside (bottom) the corotation radius (in a reference frame in which the arms are fixed).

Galactic centre. S03 argues that, as this is the present motion of the Sco-Cen stars, it favours the following star formation scenario for Sco-Cen: a shock with a spiral arm positioned slightly inside the corotation radius. However, S03 does not consider the variation in the orientation of the velocity vector of these stars from their birth to now.

From Fig. 7 we can see that, in fact, at their birth the Sco-Cen associations were moving in Galactic antirotation and away from Galactic centre. This motion is not compatible with an interaction with a spiral arm placed inside the corotation radius, and therefore contradicts the scenario proposed by S03. However, it is perfectly compatible with the expected velocity if the arm is outside the corotation radius (see Fig. 10, bottom), as recently derived in several works (see, for example, Mishurov \& Zenina 1999; and Fernández et al. 2001). Here, the difference in velocity between the shock wave and the Galactic rotation is about $2 \mathrm{~km} \mathrm{~s}^{-1} \mathrm{kpc}^{-1}$, so the difference in the velocity in the direction of Galactic rotation is about $12-13 \mathrm{~km} \mathrm{~s}^{-1}$. Following numerical simulations of the collapse of nuclei in molecular clouds (Vanhala \& Cameron 1998), one could expect a spiral arm shock wave to trigger star formation with relative velocities with respect to the gas of as low as $10 \mathrm{~km} \mathrm{~s}^{-1}$ (though the mechanism is more efficient when the velocities are between 20 and $45 \mathrm{~km} \mathrm{~s}^{-1}$ ). This scenario would then be possible in our case. Moreover, the relative velocity between the parent molecular cloud and the spiral shock wave will obviously depend on the cloud's initial velocity with respect to its regional standard of rest (RSR).

From our orbit calculations, at the moment when the molecular cloud was generating the first protostars in LCC and UCL (16-20 Myr ago, following the last estimations published in the literature), the velocity components of the cloud with respect to its RSR in the Galactic plane were $(U, V)_{\mathrm{RSR}} \sim$ $(-20,-10) \mathrm{km} \mathrm{s}^{-1}$. This motion is the result of the initial proper motion of the cloud and its interaction with a spiral shock wave ocurring about $30 \mathrm{Myr}$ ago (see Fig. 9). It is not possible to know exactly when this interaction occurred, since we do not know the value of the offset function $\Theta(R)$ for the inner arm. However, as we should expect small values for this function, we can assume that this interaction took place approximately at that epoch. 
This scenario would be in good agreement with the present ideas of rapid star formation in molecular clouds that we have presented above. The OB associations would have formed about 10-15 Myr after the arrival of the spiral shock wave, so about 15-20 Myr ago, in agreement with the estimated age for the oldest associations studied here: UCL, LCC and Tuc-Hor/GAYA (see Table 1). In this way, it is possible to explain the present observed motion of the Sco-Cen complex, and it is plausible that the origin of the star formation in the whole region was an impact with the inner spiral arm shock wave.

\subsection{The origin of YLA}

Mamajek et al. (2000) found that extrapolating past motions (assuming linear ballistic trajectories) shows that TW Hya, $\epsilon$ Cha, $\eta$ Cha and the three subgroups of the Sco-Cen complex were closest together about $10-15 \mathrm{Myr}$ ago. They suggest that these three associations were formed in the progenitor Sco-Cen GMC or in short-lived molecular clouds formed by Sco-Cen superbubbles (see also Mamajek \& Feigelson 2001). Jilinski et al. (2005) locate the birthplaces of $\epsilon$ Cha and $\eta$ Cha at the edge of LCC. Ortega et al. $(2002,2004)$ also suggest that the $\beta$ Pic moving group was formed near Sco-Cen, probably due to a supernova in this complex. Our compendium of all the known members of the whole set of YLA and the integration of their orbits back in time allow us to present here a more detailed analysis of the origin of YLA.

As we show in Sect. 3, the past trajectories of the three ScoCen associations meet in the same region of the first Galactic quadrant as YLA trajectories do. In Fig. 11 the temporal evolution of the (three-dimensional) distances between each one of the local associations and the centres of the three Sco-Cen associations is shown (with typical estimated errors of a very few tens of pc; see Sect. 3). Following these results, LCC is seen to be the association closest to YLA in the past.

The instant when the distance minima between LCC and YLA occurred is of great interest. In the cases of the $\eta$ Cha cluster and the $\epsilon$ Cha association, minima with distances of 16 and $23 \mathrm{pc}$, respectively, are obtained for $t \sim-(8.5-9)$ Myr. In the case of the $\beta$ Pic-Cap moving group, the minimum distance to LCC was 62 pc $15.5 \mathrm{Myr}$ ago. No clear distance minima to LCC are found in the recent past $(t>-20 \mathrm{Myr})$ for the other YLA. TW Hya was 45 pc from LCC 8 Myr ago (the estimated age for this association), whereas the HD 141569 system was about $102 \mathrm{pc}$ from LCC 5 Myr ago, continuously decreasing to 62 pc $18 \mathrm{Myr}$ ago. The Tuc-Hor/GAYA association has maintained a distance to LCC of more than $100 \mathrm{pc}$ over the last $20 \mathrm{Myr}$, but this could be the only YLA considered here not to originate from a supernova in LCC, since its estimated age is equal to (or even larger than) that derived for LCC.

We conclude that, at the moment of their birth, YLA were at distances of between 20 and 100 pc from the centre of LCC, and even further from the other two Sco-Cen associations (see Table 5). Although observational errors in parallax and velocity components, as well as errors in age estimations, could affect these results, the fact that we work with mean values for distances, velocity components and ages minimises this possibility. Moreover, even considering the individual stars, as they are very close, the error in the trigonometric distances derived from Hipparcos parallaxes is low for most of them (smaller than 10 pc for $89 \%$ of the stars; see Fig. 12, left). Similarly, the error in proper motions is smaller than $1.0 \mathrm{~km} \mathrm{~s}^{-1}$ for $92 \%$ of the stars (see Fig. 12, right) and errors in radial velocity of less than

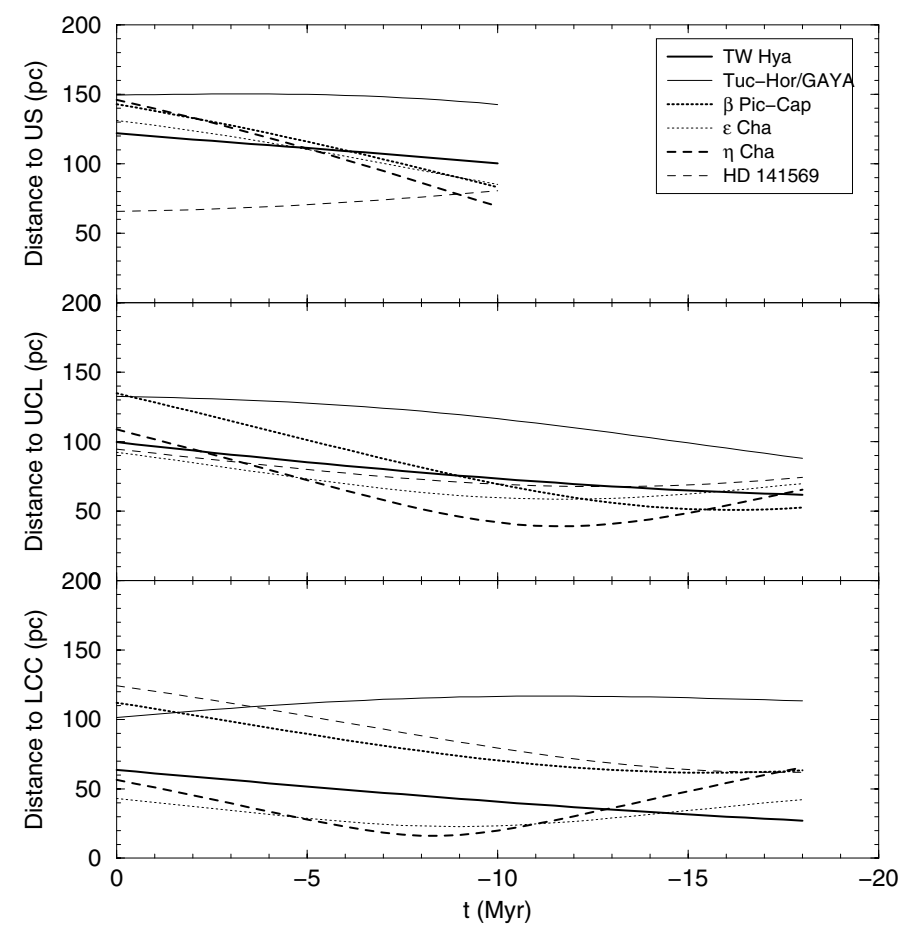

Fig. 11. Distances between the centres of YLA and the centres of the Sco-Cen associations as a function of time, using the Sco-Cen kinematics in $\mathrm{S} 03$.

Table 5. Distances (in pc) between the centres of YLA and the centres of the Sco-Cen associations at YLA birth. The distance at birth of $\beta$ PicCap to US is not shown because the latter is younger than the former.

\begin{tabular}{lcccccc}
\hline \hline Association & \multicolumn{2}{c}{ US } & \multicolumn{2}{c}{ UCL } & \multicolumn{2}{c}{ LCC } \\
& M02 & S03 & M02 & S03 & M02 & S03 \\
\hline TW Hya & 62 & 104 & 90 & 78 & 90 & 45 \\
$\beta$ Pic-Cap & - & - & 80 & 58 & 122 & 65 \\
$\epsilon$ Cha & 47 & 85 & 75 & 59 & 78 & 23 \\
$\eta$ Cha & 48 & 69 & 56 & 42 & 60 & 20 \\
HD 141569 & 43 & 70 & 85 & 80 & 120 & 102 \\
\hline
\end{tabular}

$2.0 \mathrm{~km} \mathrm{~s}^{-1}$ are estimated for most of the stars considered here (see Appendix A).

Although the present radius of LCC is about $25 \mathrm{pc}$, it has been continuously expanding since birth, 16-20 Myr ago. Even considering a very moderate expansion rate, one should expect an initial radius $\$ 20$ pc. The distances obtained in Fig. 11 and presented in Table 5, together with the expected reliability of the orbits back in time (taking into account the low associated errors and the short integration time interval), lead us to believe that the local associations were not born inside the cloud that formed the Sco-Cen complex, but in small molecular clouds outside it.

One possible scenario for the formation of YLA in these small molecular clouds is the explosion of one or several close supernovae, which could have produced compression that triggered star formation. (Lyman continuous photons from a luminous $\mathrm{O}$ star could also produce triggered star formation: see Lee 2007.) These hypothetical supernovae should belong to the Sco-Cen complex. As we have seen in Sect. 2.2, this complex is made up of several thousand stars, more than 300 of which are early-type stars, and around 35 are candidates for Type II supernovae. (They have spectral types between $\mathrm{O}$ and B2.5, and masses greater than $8 M_{\odot}$; see Weiler \& Sramek 1998; and Z99.) Maíz-Apellániz (2001) estimated the number of past 

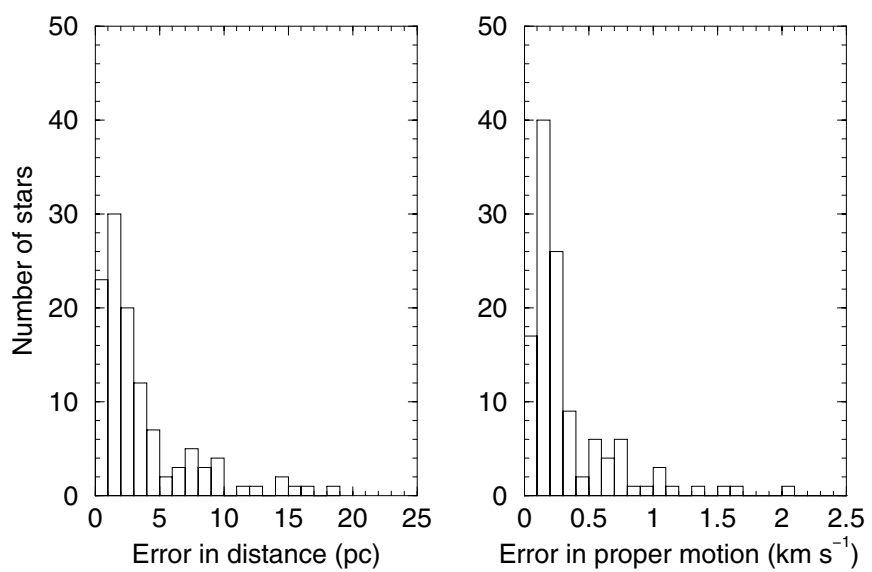

Fig. 12. Error in distance (left) and proper motion (right) for stars with Hipparcos parallaxes belonging to YLA.

supernovae inside the three associations from the Starbust99 models (Leitherer et al. 1999), obtaining 1 supernova for US, 13 for UCL and 6 for LCC. The first supernova that exploded in each association took place when it was 3-5 Myr old, and the others have been exploding and will continue to explode at a nearly constant rate, for the first $\sim 30$ Myr of the complex's life. Even a conservative estimate (considering the age for the complex proposed by de Geus et al. 1992) gives at least 6 supernovae in UCL during the last 10-12 Myr, another 6 in LCC during the last 7-9 Myr and at least 1 in US (see comments in Maíz-Apellániz 2001). There is direct observational evidence for this (Hoogerwerf et al. 2001). If the complex is slightly older (especially UCL and LCC) as stated by S03 among others, the number of supernovae in UCL and LCC in the past would increase by 5-10. It is clear that there have been several recent supernovae in the solar neighbourhood, although it is necessary to know where the Sco-Cen associations were in the past to see if they can be linked to the origin of YLA presented in Sect. 2.3. Figures 7 and 8 also show the orbits of US, UCL and LCC back to their birth epochs.

The wave front of a supernova typically moves at a velocity of a few tens of pc per million years ${ }^{4}$; therefore, a supernova explosion in LCC or UCL 9-11 Myr ago may have triggered star formation between 1 and 3 Myr later in small molecular clouds at distances of 15 to $75 \mathrm{pc}$. These would be the parent clouds of the $\eta$ Cha cluster and the $\epsilon$ Cha association, located at $\sim 20 \mathrm{pc}$ from LCC at their birth. Taking into account that the first supernovae in LCC and UCL exploded when the associations were 3-5 Myr old (Maíz-Apellániz 2001), this scenario is only possible for a present age of LCC and UCL of at least 12 Myr. This is not a problem, since the estimated ages for LCC published in the literature range from 11-12 Myr to 16-20 Myr, and for UCL, from $14-15$ to $16-20 \mathrm{Myr}$, as we have seen. In this way, if only one supernova could explain this star formation outbreak $\sim 8.5-$ 9 Myr ago, this would be the age of $\eta$ Cha and $\epsilon$ Cha, which would have been formed simultaneously. It should be remembered that the estimated ages for $\eta$ Cha and $\epsilon$ Cha are 5-15 and $\$ 10$ Myr, respectively (see Table 1).

\footnotetext{
${ }^{4}$ After considerable slowing during the first 3-5 pc of distance covered, the wave front of a typical supernova moves at $\sim 15-45 \mathrm{~km} \mathrm{~s}^{-1}$ when it is at a distance of $\sim 10-100$ pc from the explosion site (see Vanhala \& Cameron 1998; and Preibisch et al. 2002). A velocity of $30 \mathrm{~km} \mathrm{~s}^{-1}$ is equal to $30.7 \mathrm{pc} \mathrm{Myr}^{-1}$.
}

Such a supernova could also have triggered the formation of TW Hya, whose estimated age is $\sim 8$ Myr. As mentioned above, at that time TW Hya was about 45 pc from the centre of LCC, in perfect agreement with the typical distance at which a supernova wave front can trigger star formation in a small molecular cloud. However, it was not necessarily a single supernova in LCC or UCL that was the origin of these four YLA. The supernova rate in these two associations is $\sim 0.5 \mathrm{Myr}^{-1}$ and, therefore, it is possible that a few supernovae in the period $-8 \lesssim t \lesssim-10 \mathrm{Myr}$ triggered the star formation that resulted in YLA. In any case, from our results we can conclude that these associations definitely did not form inside LCC or UCL, to be later ejected. They were formed in regions of space far from LCC and UCL, probably in small molecular clouds that were later totally dispersed by the newly born stars and/or by the shock fronts of later supernovae in LCC or UCL.

Our results support a star formation scenario for very young stars far away from SFR or molecular clouds, such as that proposed by Feigelson (1996) and not that of Sterzik \& Durisen (1995). The latter authors perform numerical simulations to explain the existence of haloes of isolated T Tau stars around SFR. In their simulations a significant number of stars were ejected from these regions at birth with large velocities, allowing trajectories of some tens of pc in a few million years. Meanwhile, Feigelson (1996) proposed another scenario for the formation not only of the haloes of T Tau stars, but also of other completely isolated very young stars that have been discovered. In this model, the isolated T Tau stars form in small, fast-moving, short-lived molecular clouds. The gas remaining after the star formation process is rapidly dispersed by the stellar winds of the new stars. At present the stars are located in regions of space where there is no gas and so, apparently, they have formed far away from any SFR. The case of YLA supports this scenario, since our kinematic study shows that these associations formed far away from the Sco-Cen complex. For the HD 141569 system, a supernova in UCL, as opposed to one in LCC, is a more promising candidate to explain its origin. This is because the distance to LCC for the range of ages accepted for this group (2-8 Myr) is between 88 and 116 pc, whereas for UCL it is 7388 pc.

\subsection{YLA and $L B$}

Several models have been presented to explain the origin of the LB. Cox (1998) reviews five conceptions of the LB on which models have been based. Most of them involve one or more supernovae. The consensus is reached with a scenario in which about 10-20 supernovae formed the local cavity and, after that, a few supernovae reheated the LB a few Myr ago, explaining the currently observed temperature of the SXRB (Breitschwerdt \& Cox 2004). Some authors have remarked that there is independent evidence for the occurrence of a close supernova ( $30 \mathrm{pc})$ $\sim 5$ Myr ago (Knie et al. 1999), which could be the best candidate for reheating the LB. Smith \& Cox (2001) find that one of the problems with this theory is the low probability of having 2 or 3 isolated supernovae exploding in the solar neighbourhood in a period of a few million years. In an attempt to solve this problem, Maíz-Apellániz (2001) proposed that the 2 or 3 supernovae that reheated the local cavity (and which are therefore responsible for the LB) could have exploded in LCC, but his results faced some geometrical problems due to the peripheral situation of LCC with respect to the LB. Berghöfer \& Breitschwerdt (2002) suggest that the supernovae came from the B1 moving group found by Asiain et al. (1999a), comprised of 33 stars with 


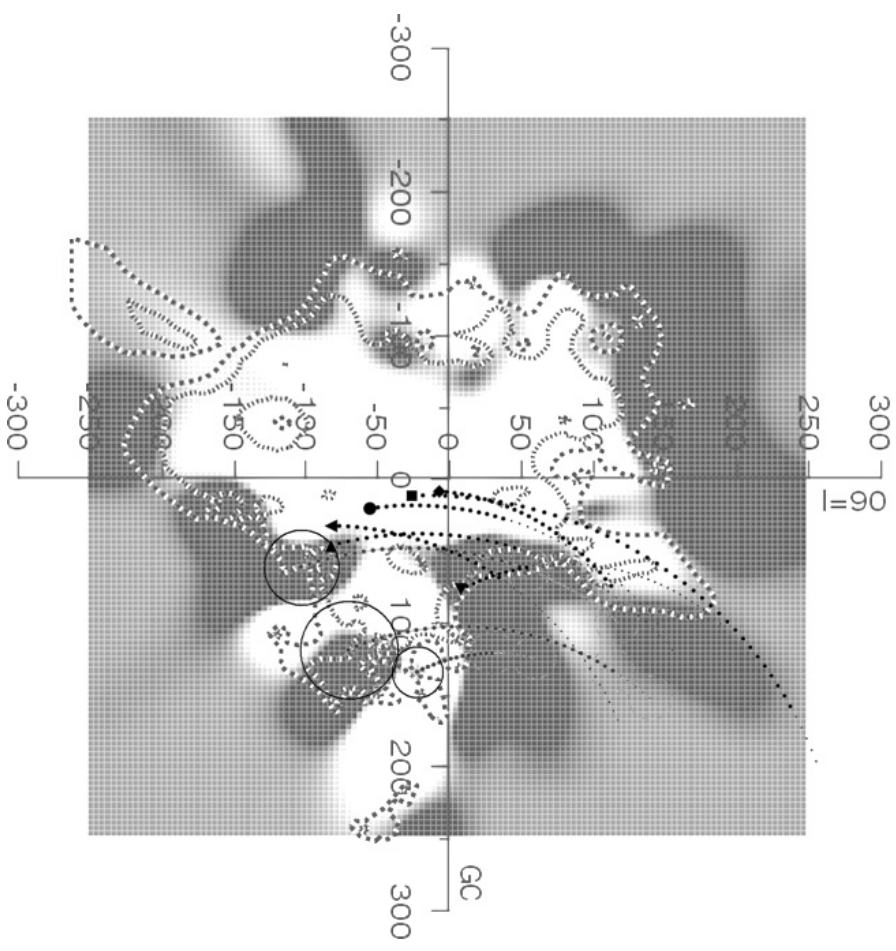

Fig. 13. Orbits of YLA and the Sco-Cen associations back in time to their individual ages (in a system comoving with the LSR; see Fig. 7) superimposed on the LB structure from Lallement et al. (2003, see Fig. 1). This structure has been rotated $90^{\circ}$ with respect to the orientation shown in Fig. 1.

spectral types B, A and F. However, the mean distance to the Sun (and so to the centre of the LB) of its members is $\sim 135 \mathrm{pc}$ and the trajectory back in time of the B1 group passes through very peripheral zones of the LB (in fact, the B1 group is formed by stars in the Sco-Cen complex).

More recently, Fuchs et al. (2006) have proposed that about 14-20 SNe, originated from the LCC and UCL associations, have been excavating the LB during the last $\sim 14$ Myr. The authors argue that this scenario is realistic even though the $\mathrm{SNe}$ exploded rather close to the edge of the LB, since the bubble expands fastest in the direction of the lowest density regions. In the present case, the presence of the Loop I in the GC direction would forced the LB to expand towards the anticentre direction. However, part of the geometric problems that these works face could be overcome by assuming that one or more recent supernovae exploded inside YLA. In Fig. 13 we show the trajectories back in time for YLA, superimposed on the present LB structure derived by Lallement et al. (2003). Due to small peculiar motions of the local interstellar gas, the LB is assumed to be nearly at rest in the reference frame of the LSR (as done by Fuchs et al. 2006). Thus, the position of the centre of the LB is fixed in time at the system comoving with the LSR (where the orbits have been computed; see Fig. 13). As the LB has been in expansion during the last Myr, the outer boundaries were placed nearer to its centre in the past. Due to the uncertainties of the present outer boundary of the LB and the wealth of data, most models have shortcomings concerning the dynamical evolution of the structure (Breitschwerdt \& de Avillez 2006). Even when not considering the dynamical evolution of the LB (i.e., the expansion motion of the boundary), we are able to obtain important conclusions when comparing the past trajectories of both YLA and Sco-Cen associations. In Fig. 13 we can see that the past orbits of the YLA are closer to the central region of the LB than
Sco-Cen associations. To be exact, the trajectories of the centres of the associations TW Hya, Tuc-Hor/GAYA and $\beta$ Pic-Cap have crossed very near to the geometric centre of the LB in the last $\sim 5$ Myr. As can be seen in the error propagation of the kinematic data shown in Fig. 7, the uncertainty boxes in position (due to the errors in the mean velocity vector) on the Galactic plane do not exceed a few tens of pc. On the other hand, errors in age estimates for the YLA result in positional errors along the trajectories back in time. Even considering the large uncertainties in age obtained for some of these YLA (see Table 2), ages are not expected to exceed $20 \mathrm{Myr}$ for any of them (except for TucHor/GAYA). We can therefore conclude that the YLA have been moving inside the LB for (at least) most of their lifetime, and can question whether the presence of these young stars inside the LB bears any relation to its origin and/or evolution.

Table 6 gives the spectral types of the known members of the local associations. All the associations except TW Hya and $\beta$ Pic-Cap contain B-type stars (13 stars from a total of 223 members). At present it is not possible to derive the number of stars earlier than B2.5 that were born in the local associations, since we do not know their total mass precisely. However, the fact that at present we observe one supernova candidate ( $\alpha$ Pav, a B2IV star belonging to the Tuc-Hor/GAYA association), and more than a dozen stars of spectral types between B5 and B9, allows us to affirm that it is possible that one or more of these associations has sheltered a supernova in the recent past (the last $10 \mathrm{Myr}$ ). As there is direct evidence for an explosion of a supernova at a distance of $\sim 30 \mathrm{pc}, \sim 5 \mathrm{Myr}$ ago, several pieces of the same puzzle seem to support the theory of a recent supernova in the nearest solar neighbourhood originating from a parent star belonging to a YLA, probably Tuc-Hor/GAYA or the extended R $\mathrm{CrA}$ association (which currently show the highest content of B-type stars). This near and recent supernova would have been responsible for the reheating of the gas inside the LB needed to achieve the currently observed temperature of the diffuse soft X-ray background.

As we mention above, there is no agreement in the literature on the number of supernovae needed to form the local cavity. If only one was enough, the supernova we propose would be the most promising candidate, since it would be placed very near the geometric centre of the LB, explaining in a natural way its present spatial structure. If more supernovae are needed (as recent works suggest), we could consider other stars in the vicinity of LCC and UCL, as proposed by Fuchs et al. (2006).

\section{Our scenario}

If the impact of the spiral arm shock wave was the initial cause of star formation in the Sco-Cen region (see Sect. 4.1) then the history of the nearest solar neighbourhood during the last few tens of millions of years would have been as follows. $30 \mathrm{Myr}$ ago the GMC that became the parent of Sco-Cen was in the Galactic plane with coordinates $\left(X^{\prime}, Y^{\prime}\right) \sim(-400,1200)$ pc (see Fig. 9). The arrival of the potential minimum of the inner spiral arm triggered star formation in the region. At the same time it disturbed the cloud's motion, whose velocity vector became directed in the opposite direction to Galactic rotation and away from Galactic centre (just as expected after an interaction with the spiral arm for a position outside the corotation radius; see Fig.10, bottom). The compression due to the spiral arm did not necessarily trigger star formation in the whole cloud, but perhaps only in the regions with the largest densities. This would be favoured by the smaller relative velocity between the shock wave and the RSR (12-13 $\mathrm{km} \mathrm{s}^{-1}$, as we have seen). The regions 
Table 6. Spectral type of known YLA members.

\begin{tabular}{|c|c|c|c|c|c|c|c|c|c|c|c|c|c|c|c|}
\hline Association & B2-B4 & B5-B9 & A0-A4 & A5-A9 & F0-F4 & F5-F9 & G0-G4 & G5-G9 & K0-K4 & K5-K9 & M0-M2 & M3-M5 & M6-M9 & $?$ & Total \\
\hline TW Hya & & & 1 & & & & & 1 & 2 & 6 & 17 & 3 & 2 & 7 & 39 \\
\hline Tuc-Hor/GAYA & 2 & 3 & 5 & 2 & 4 & 9 & 2 & 5 & 8 & 4 & 2 & 2 & & 4 & 52 \\
\hline$\beta$ Pic-Cap & & & 3 & 2 & 2 & 5 & 2 & & 3 & 4 & 4 & 6 & 1 & 1 & 33 \\
\hline$\epsilon$ Cha & & 1 & 1 & 1 & & 1 & & 1 & 5 & & 4 & 1 & & 1 & 16 \\
\hline$\eta$ Cha & & 1 & & 3 & & & & & 3 & & 4 & 8 & & & 19 \\
\hline HD 141569 & & 1 & 2 & & & & & & & & 1 & 1 & & & 5 \\
\hline Ext. R CrA & & 5 & & 1 & 1 & 1 & 1 & 4 & 14 & 7 & 8 & 15 & 1 & 1 & 59 \\
\hline Total & 2 & 11 & $\overline{12}$ & 9 & 7 & 16 & 5 & 11 & 35 & 21 & 40 & 36 & 4 & 14 & 223 \\
\hline
\end{tabular}

where star formation began must be those which generated UCL, LCC and, probably, the Tuc-Hor/GAYA association, which were all born at nearly the same time: about 16-20 Myr ago. The stellar winds from the first massive stars began to compress the gas of the neighbouring regions, maybe causing them to fragment into small molecular clouds that moved away from the central region of the parent cloud. About 9 Myr ago, a supernova in LCC or UCL triggered star formation in these small molecular clouds, giving birth to the majority of YLA, as we saw in the previous section. The stellar winds of the newly born stars rapidly expelled the remaining gas from these small clouds (the cloudlets proposed by Feigelson 1996), completely erasing every trace of them and leading to our observation that there is no gas in these regions at present. YLA may have had a crucial influence on the history of the LB. We suggest that one or two supernovae in these associations were responsible for reheating the LB a few million years ago. This hypothesis seems to be reinforced by the evidence of a very near supernova about $5 \mathrm{Myr}$ ago (Knie et al. 1999). At about the same time, as proposed by Preibisch \& Zinnecker (1999), the shock front of a supernova in UCL would have triggered star formation in US about $6 \mathrm{Myr}$ ago. Only 1.5 Myr ago, the most massive star in US would have gone supernova and its shock front would now be reaching the molecular cloud of $\rho$ Oph, triggering the beginning of the star formation process there.

\section{Conclusions}

This paper studies the kinematic evolution of the Sco-Cen complex and the so-called young local associations (YLA). It makes use of most of the astrophysical data published in the literature for all the known members of YLA (more than 200 stellar systems). This information appears in Appendix A and can also be accessed via a webpage ${ }^{5}$.

The study of the orbits integrated back in time for all these associations allows us to propose a scenario for recent star formation in the solar neighbourhood (Sect. 5) and a possible link between these associations and the origin and/or evolution of the LB (Sect. 4.3). In our scenario, the oldest Sco-Cen associations were brought about by the impact of the inner spiral arm against a giant molecular cloud. YLA were born later (and outside ScoCen) due to the shock fronts of the most massive supernovae belonging to the LCC or UCL associations. Our results suggest that a YLA is the most likely place to have harboured the supernova that reheated the LB a few million years ago.

As seen in this paper, the recent discovery of a set of YLA, together with the use of appropriate tools (mainly, the integration back in time of orbits), sheds light on several apparently unrelated topics in astrophysics. These topics range from star formation mechanisms for low-mass stars distant from star forming

5 http://www.am.ub.es/ dfernand/YLA/ regions, to the recent history of the $\mathrm{LB}$, and include the origin of the Sco-Cen stellar complex and its possible independence from the Gould Belt. The possible discovery in the coming years of new members of these associations, or even of new associations, will be very useful in confirming the results obtained in this work.

Acknowledgements. This work was supported by the CICYT under contracts AYA2003-07736 and AYA2006-15623-C02-02.

\section{Appendix A: Young local associations}

This Appendix is a compendium of present knowledge about YLA that have been discovered over the last decade. The information contained in the tables can be accessed via a webpage ${ }^{6}$.

\section{A.0.1. The TW Hya association}

TW Hya has been the prototype of isolated T Tau stars since its classification as a classical T Tau star (CTTS) of about $10 \mathrm{Myr}$ by Rucinsky \& Krautter (1983). It is far away from any dark cloud and even from other PMS stars. Gregorio-Hetem et al. (1992) identified four candidate T Tau stars in a circle of $10^{\circ}$ around TW Hya. The existence of a real association (the socalled TW Hya association or TWA) was finally established when Webb et al. (1999) found seven new members, including a brown dwarf. These new members showed important X-ray emissions, a large abundance of $\mathrm{Li}$, intense chromospheric activity and a proper motion similar to that observed in the previously identified members of TWA. Webb et al. (1999) derived an age of $8 \mathrm{Myr}$ for the association from the colour-magnitude diagram. Barrado y Navascués (2006), making use of the location in the HR diagram and $\mathrm{H} \alpha$ and $\mathrm{Li}$ equivalent widths for individual stars, estimated an age of $10_{-7}^{+10} \mathrm{Myr}$.

Sterzik et al. (1999), Zuckerman et al. (2001c), Makarov \& Fabricius (2001), Gizis (2002), Reid (2003) and Song et al. (2003) added more stars to the list, increasing the number of members to 25 stellar systems. Table 7 is adapted from Torres et al. (2003) and shows the members of TWA, with the addition of the members identified by Reid (2003) and Song et al. (2003).

\section{A.0.2. The Tuc-Hor/GAYA association}

The Tucana and Horologium associations were discovered by Zuckerman \& Webb (2000) and Torres et al. (2000), independently. The former authors performed a search of comoving stars around IRAS sources, whereas Torres et al. searched for ROSAT sources around the active star ER Eri. Zuckerman et al. (2001a) proposed merging the two associations into one, the socalled Tuc-Hor association, due to their proximity in the sky and the similarities in their velocity components and age. Later,

\footnotetext{
${ }^{6}$ http://www.am.ub.es/ dfernand/YLA/
} 
Torres et al. (2001) proposed the denomination GAYA association for the stars that belonged to the Tuc-Hor association and some other young stars in a region of the sky with an angular extent of about $60^{\circ}$. Song et al. (2003) found new members of the association.

Table 8 shows the members of the Tuc-Hor/GAYA association. The first two sections of the Table show the data for the stars originally assigned to the Tuc association (nucleus and halo of the group). The stars originally assigned to the Hor association are shown in Sects. 3 to 5 (probable and possible members, and early-type stars). The sixth section includes the stars proposed by Zuckerman et al. (2001a) and the seventh section lists the stars identified as members by Song et al. (2003).

\section{A.0.3. The $\beta$ Pic-Cap moving group}

Since Smith \& Terrile (1984) identified a dust disk around $\beta$ Pic, determining the age for this A5V star has been a major goal in the field of planet formation. Barrado y Navascués et al. (1999) used the list of possible kinematic companions of $\beta$ Pic to investigate the existence of a real moving group that could provide clues to the age of this star. Using accurate radial velocities, proper motions and parallaxes, Barrado $y$ Navascués et al. (1999) found that 6 stars share the motion of $\beta$ Pic, but only 2 of them have a common age. Zuckerman et al. (2001a) searched for members of the $\beta$ Pic moving group in the Hipparcos catalogue and found 18 candidate stellar systems (including some members previously assigned to the Tucana association by Zuckerman \& Webb 2000; and to the Capricornus association by van der Ancker et al. 2000, 2001) with a mean heliocentric distance of $35 \mathrm{pc}$. This mean distance meant that $\beta$ Pic was the nearest moving group to the Sun (though a few years later this honour passed to the AB Dor moving group). The age of the group determined by Zuckerman et al. (2001a) is about 12 Myr. Song et al. (2003) added new members to the group, which has a spatial extent of about $100 \mathrm{pc}$.

Ortega et al. (2002) studied the orbits back in time of the members of the $\beta$ Pic-Cap moving group and found a kinematic age of 11.5 Myr. At that time the group was at a distance of about 45 pc from the centre of the LCC and UCL complexes in ScoCen. The authors believe that an explosion in the periphery of LCC or UCL 12.5 Myr ago could have triggered the formation of the $\beta$ Pic group 1 Myr later.

Table 9 lists the known members of the $\beta$ Pic-Cap moving group: in the first section, there are those stars identified by Zuckerman et al. (2001a) and in the second section, those selected by Song et al. (2003).

\section{A.0.4. The $\epsilon$ Cha association}

The Chamaeleon cloud complex was first studied by Hoffmeister (1962), who identified several RW Aur-type variable stars, some of them showing $\mathrm{H} \alpha$ emission. Before the ROSAT mission, it was suspected that 14 stars were connected to the Chamaeleon region. The optical counterparts of the ROSAT sources identified several dozen new candidate PMS stars (Alcalá et al. 1995, 1997). Thirty of them were confirmed through Li abundance calculations (Covino et al. 1997).

The so-called $\epsilon$ Cha association was discovered by Frink et al. (1998; see also Frink 1999), studying the kinematics of $\mathrm{T}$ Tau stars located not only in the cloud nuclei, but also in regions far away from them. Mamajek et al. (2000) added some stars detected by Terranegra et al. (1999) and Eggen (1998) to the group.

Table 10 lists the known members of the $\epsilon$ Cha association, with data from Frink et al. (1998) Terranegra et al. (1999) and Mamajek et al. (2000).

\section{A.0.5. The $\eta$ Cha cluster}

The $\eta$ Cha cluster was discovered by Mamajek et al. (1999) from observations made in 1997 using the High Resolution Imager (HRI) of the ROSAT satellite. ROSAT had previously detected four X-ray sources around the star $\eta \mathrm{Cha}$, which were identified as weak-line T Tau stars (Alcalá et al. 1995; Covino et al. 1997). From the HRI observations, $12 \mathrm{X}$-ray sources were discovered in an area of $0.2 \mathrm{sqr}$ deg, each one with a prominent optical counterpart. Spectroscopic observations of these stars were made by Mamajek et al. (1999) and led to the stars being classified as PMS stars belonging to the $\eta$ Cha cluster. The brightest stars in the cluster have similar parallaxes and proper motions in the Hipparcos catalogue, confirming the cluster's identity. Lawson (2001) and Lawson et al. (2002) discovered two new members of the cluster, while Song et al. (2004), Ran Lyo et al. (2004) and Luhman \& Steeghs (2004) independently added three new members, giving a total of 18 stellar systems with 9 secondary stars.

Table 11 lists the known members of the $\eta$ Cha cluster. It should be complete down to $0.15 M_{\odot}$ (or spectral type M6; see Ran Lyo et al. 2004) or even $0.015 M_{\odot}$ (Luhman \& Steeghs 2004).

\section{A.0.6. The HD 141569 system}

Weinberger et al. (2001) searched around the triple star HD 141569 Hipparcos for candidates sharing distance and motion with this system. They found that two stars of spectral type A lie below or along the ZAMS, where the young A-type stars are placed.

Table 12 lists the members of the HD 141569 system; a total of 3 stellar systems with 5 stars.

\section{A.0.7. The extended R CrA association}

In the solar neighbourhood, the densest nucleus of a molecular cloud is the dark cloud near the star R CrA (Dame et al. 1987), which has an extinction of up to $45 \mathrm{mag}$ in the visible range. Several IR surveys have shown the existence of a large population of IR sources inside the cloud (see, for example, Taylor \& Storey 1984; or Wilking et al. 1997). The age of the cloud has been estimated at between 1 Myr (Knacke et al. 1973) and 6 Myr (Wilking et al. 1992). Marraco \& Rydgren (1981) obtained a distance of about $129 \mathrm{pc}$ for the cloud. The large error in the Hipparcos parallax for the star R CrA did not shed new light on this issue.

Since the 1970s, a few classical T Tau stars have been associated with the CrA dark cloud (see for example Knacke et al. 1973; Wilking et al. 1986, 1992). More recently, Neuhäuser et al. (2000) conducted an optical identification program between the non-identified RASS (ROSAT All-Sky Survey) sources to find PMS stars in and around the CrA dark cloud. They found 19 optical counterparts that fulfil PMS star conditions; 2 of them are classical T Tau stars, whereas the other stars are weak-line T Tau stars. The spatial distribution of these stars does not show the proximity to the cloud observed in the former members. Another 
21 possible members were found by Quast et al. (2001) in a region of the sky with a projected diameter of around $35^{\circ}$. This fact led the authors to rename the group extended $\mathrm{R} C r A$ association.

Table 13 shows the known members of the extended R CrA association. The two first sections include those PMS stars known before the ROSAT mission and 4 B-type stars that could also be associated with the CrA cloud. The third section lists the 19 new members, all of them T Tau stars, discovered by Neuhäuser et al. (2000). The last section shows the two new members explicitly mentioned by Quast et al. (2001) among the 21 new members that they found.

\section{A.0.8. The AB Dor moving group}

Zuckerman et al. (2004) identified about 40 nearby stars that are moving through space together with the intensively studied star $\mathrm{AB}$ Dor. The mean distance to these stars is $32 \mathrm{pc}$, making it the closest group to Earth. The authors performed spectroscopic observations and derived radial velocities, rotational velocities and equivalent widths of the $\mathrm{H} \alpha$ and $\mathrm{Li} \lambda 6708$ lines. They estimated the age of the moving group as $50 \mathrm{Myr}$, comparing the $\mathrm{H} \alpha$ emission and absorption of the late $\mathrm{K}$ - and early M-type stars in the AB Dor moving group with those identified in the Tucana association. Luhman et al. (2005) estimated an age in the range 75-150 Myr using colour-magnitude diagrams. This would make the moving group roughly coeval with the Pleiades ( $\tau$ 100-125 Myr; see, for example, Stauffer et al. 1998; and Meynet et al. 1993) and therefore much older than the other associations studied in this section. However, López-Santiago et al. (2006) found that the moving group can be split into two subgroups, with ages of 30-50 Myr and 80-120 Myr. On the other hand, Makarov (2007) proposes that the nucleus of the AB Dor moving group formed $38 \mathrm{Myr}$ ago during a close passage of the Cepheus OB6 cloud. In any case, we can see that an age greater than $30 \mathrm{Myr}$ is proposed in all works.

Table 14 shows the members of the AB Dor moving group found by Zuckerman et al. (2004). The first section of the table includes the more probable members, and the second, shows stars whose membership of the moving group is questionable.

\section{References}

Alcalá, J. M., Krautter, J., Schmitt, J. H. M. M., et al. 1995, A\&AS, 114, 109

Alcalá, J. M., Krautter, J., Covino, E., et al. 1997, A\&A, 319, 184

Allen, C., \& Santillán, A. 1991, Rev. Mex. Astron. Astrofis., 22, 255

Amôres, E. B., \& Lépine, J. R. D. 2005, AJ, 130, 659

van den Ancker, M. E., Pérez, M. R., de Winter, D., \& McCollum, B. 2000, A\&A, 363, L25

van den Ancker, M. E., Pérez, M. R., \& de Winter, D. 2001, in Young stars near Earth: Progress and Prospects, ed. R. Jayawardhana, T. P. Greene, ASP Conf. Ser. 244, 69

Andersson, B.-G., Idzi, R., Uomoto, A., et al. 2002, AJ, 124, 2164

Asiain, R. 1998, Ph.D. Thesis, Universitat de Barcelona, Spain

Asiain, R., Figueras, F., Torra, J., \& Chen, B. 1999a, A\&A, 341, 427

Asiain, R., Figueras, F., \& Torra, J. 1999b, A\&A, 350, 434

Barrado y Navascués, D. 2006, A\&A, 459, 511

Barrado y Navascués, D., Stauffer, J. R., Song, I., \& Caillaut, J.-P. 1999, ApJ, 520, L123

Berghöfer, T. W., \& Breitschwerdt, D. 2002, A\&A, 390, 299

Bertin, G., Lin, \& C. C. 1996, Spiral Structure in Galaxies: A Density Wave Theory (Cambridge: The MIT Press)

Binney, J., \& Merrifield, M. 1998, Galactic Astronomy (Princeton: Princeton University Press)

Binney, J., Gerhard, O. E., Stark, A. A., Bally, J., \& Uchida, K. I. 1991, MNRAS, 252,210

Blaauw, A. 1960, in Present Problems Concerning the Structure and Evolution of the Galactic System, ed. J. H. Oort, \& H. G. Quik, Nuffic Intern. Summer Course, 3,1
Blaauw, A. 1964, ARA\&A, 2, 213

Blaauw, A. 1991, in The Physics of Star Formation and Early Stellar Evolution, ed. C. J. Lada, \& N. D. Kylafis, NATO ASI Ser. C, 342, 125

Breitschwerdt, D., \& Cox, D. P. 2004, in How does the Galaxy work? A Galactic Tertulia with Don Cox and Ron Reynolds, ed. E. J. Alfaro, E. Pérez, \& J. Franco, Astrophysics and Space Science Library, 315, 391

Breitschwerdt, D., \& de Avillez, M. A. 2006, A\&A, 452, L1

Chauvin, G., Thomson, M., Dumas, C., et al. 2003, A\&A, 404, 157

Clark, P. C., Bonnell, I. A., Zinnecker, H., \& Bate, M. R. 2005, MNRAS, 359, 809

Covino, E., Alcalá, J. M., Allain, S., et al. 1997, A\&A, 328, 187

Cox, D. P. 1998, in The Local Bubble and Beyond, ed. D. Breitschwerdt, M. J. Freyberg, \& J. Trümper, Lecture Notes in Physics, 506, Proc. IAU Colloq., 166,121

Dame, T. M., Ungerechts, H., \& Cohen, R. S. 1987, ApJ, 322, 706

Dobbs, C. L., \& Bonnell, I. A. 2007, MNRAS, 376, 1747

Eggen, O. J. 1961, R. Obs. Bull., 41, 245

Eggen, O. J. 1965a, in Galactic structure, ed. A. Blaauw, \& M. Schmidt (Univ. Chicago Press), 111

Eggen, O. J. 1965b, ARA\&A, 3, 235

Eggen, O. J. 1998, private communication

Feigelson, E. D. 1996, ApJ, 468, 306

Feigelson, E.D., Lawson, W. A., \& Garmire, G. P. 2003, ApJ, 599, 1207

Fernández, D. 2005, Ph.D. Thesis, Universitat de Barcelona, available from http: //tdx. cesca.es/TDX-0316105-114904/

Fernández, D., Figueras, F., \& Torra, J. 2001, A\&A, 372, 833

Frink, S. 1999, Ph.D. Thesis, Universität Heidelberg

Frink, S., Röser, S., Alcalá, J. M., Covino, E., \& Brandner, W. 1998, A\&A, 338, 442

Fuchs, B., Breitschwerdt, D., de Avillez, M. A., Dettbarn, C., \& Flynn, C. 2006, MNRAS, 373, 993

Georgelin, Y. M., \& Georgelin, Y. P. 1976, A\&A, 49, 57

de Geus, E. 1992, A\&A, 262, 258

de Geus, E., de Zeeuw, P. T., \& Lub, J. 1989, A\&A, 216, 44

Gittins, D. M., \& Clarke, C. J. 2004, MNRAS, 349, 909

Gizis, J. E. 2002, ApJ, 575, 484

Gregorio-Hetem, J., Lépine, J. R. D., Quast, G. R., Torres, C. A. O., \& de la Reza, R. 1992, AJ, 103, 549

Hearty, T., Neuhäuser, R., Stelzer, B., et al. 2000a, A\&A, 353, 1044

Hearty, T., Fernández, M., Alcalá, J. M., Covino, E., \& Neuhäuser, R. 2000b, A\&A, 357, 681

Hoffmeister, C. 1962, Z. Astrophys., 55, 290

Hoogerwerf, R., de Brujine, J. H. J., \& de Zeeuw, P. T. 2001, A\&A, 365, 49

Jayawardhana, R. 2000, Science, 288, 64

Jilinski, E., Ortega, V. G., \& de la Reza, R. 2005, ApJ, 619, 945

Kerr, F. J., \& Lynden-Bell, D. 1986, MNRAS, 221, 1023

Knie, K., Korschinek, G., Faestermann, T., et al. 1999, Phys. Rev. Lett., 81, 18

Knacke, R. F., Strom, K. M., Strom, S. E., Young, E., \& Kundel, W. 1973, ApJ, 179,847

Kurucz, R. L. 1993, ATLAS9 Stellar Atmosphere Programs and $2 \mathrm{~km} / \mathrm{s}$ grid. Kurucz CD-ROM No. 13, Cambridge, Massachussets, Smithsonian Astrophysical Observatory

Lallement, R., Welsh, B. Y., Vergely, J. L., Crifo, F., \& Sfeir, D. M. 2003, A\&A, 411,447

Landau, L. D., \& Lifshitz, E. M. 1982, Fluid Mechanics (Oxford: Pergamon Press)

Lawson, W. A., Crause, L. A., Mamajek, E. E., \& Feigelson E. D. 2001, MNRAS, 321, 57

Lawson, W. A., Crause, L. A., Mamajek, E. E., \& Feigelson E. D. 2002, MNRAS, 329, L29

Lee, H.-T., \& Chen, W. P. 2007, ApJ, 657, 884

Leitherer, C., Schaerer, D., Goldader, J. D., et al. 1999, ApJS, 123, 3

Lin, C. C., \& Shu, F. H. 1964, ApJ, 140, 646

Lin, C. C., Yuan, C., \& Shu, F. H. 1969, ApJ, 155, 721

Lindblad, P. O. 1967, Bull. Astron. Inst. Netherlands, 19, 34

López-Santiago, J., Montes, D., Crespo-Chacón, I., \& Fernández-Figueroa, M. J. 2006, ApJ, 643, 1160

Lowrance, P. J., McCarthy, C., Becklin, E. E., et al. 1999, ApJ, 512, L69

Luhman, K. L. 2001, ApJ, 560, 287

Luhman, K. L., \& Steeghs, D. 2004, ApJ, 609, 917

Luhman, K. L., Stauffer, J. R., \& Mamajek, E. E. 2005, ApJ, 628, L69

Madsen, S., Dravins, D., \& Lindegren, L. 2002, A\&A, 381, 446 [M02]

Maíz-Apellániz, J. 2001, ApJ, 560, 83

Makarov, V. V. 2007, ApJS, 169, 105

Makarov, V. V., \& Fabricius, C. 2001, A\&A, 368, 866

Makarov, V. V., Gaurne, R. A., \& Andrievsky, S. M. 2005, MNRAS, 362, 1109

Mamajek, E. E. 2005, ApJ, 634, 1385 
Mamajek, E. E., \& Feigelson, E. D. 2001, in Young stars near Earth: Progress and Prospects, ed. R. Jayawardhana, \& T. P. Greene, ASP Conf. Ser., 244, 104

Mamajek, E. E., Lawson, W. A., \& Feigelson, E. D. 1999, ApJ, 516, L77

Mamajek, E. E., Lawson, W. A., \& Feigelson, E. D. 2000, ApJ, 544, 356

Mamajek, E. E., Meyer, E. D., \& Liebert, J. 2002, AJ, 124, 1670

Marraco, H. G., \& Rydgren, A. E. 1981, AJ, 86, 62

Merín, B., Montesinos, B., Eiroa, C. et al. 2004, A\&A, 419, 301

Meynet, G., Mermilliod, J.-C., \& Maeder, A. 1993, A\&AS, 98, 477

Mishurov, Yu.N., \& Zenina, I. A. 1999, A\&A, 341, 81

Miyamoto, M., \& Nagai, R. 1975, PASJ, 27, 533

Moreno, E., Alfaro, E. J., \& Franco, J. 1999, ApJ, 522, 276

Neuhäuser, R., \& Brandner, W. 1998, A\&A, 330, L29

Neuhäuser, R., Walter, F. M., Covino, E. et al. 2000, A\&AS, 146, 323

Olano, C. A. 1982, A\&A, 112, 195

Ortega, V. G., de la Reza, R., Jilinski, E., \& Bazzanella, B. 2002, ApJ, 575, L75

Ortega, V. G., de la Reza, R., Jilinski, E., \& Bazzanella, B. 2004, ApJ, 609, 243

Ortega, V. G., Jilinski, E., de la Reza, R., \& Bazzanella, B. 2007, MNRAS, 377, 441

Palouš, J., Jungwiert, B., \& Kopecký, J. 1993, A\&A, 274, 189

Paresce, F. 1984, AJ, 89, 1022

Pöppel, W. G. L. 1997, The Gould Belt System and the Local Interstellar Medium, Fundamental of Cosmic Physics, 18

Preibisch, T., \& Zinnecker, H. 1999, AJ, 117, 2381

Preibisch, T., Brown, A. G. A., Bridges, T., Guenther, E., \& Zinnecker, H. 2002, AJ, 124, 404

Quast, G. R., Torres, C. A. O., de la Reza, R., da Silva, L., \& Drake, N. 2001, in Young stars near Earth: Progress and Prospects, ed. R. Jayawardhana, \& T. P. Greene, ASP Conf. Ser., 244, 49

Ran Lyo, A., Lawson, W. A., Feigelson, E. D., \& Crause L. A. 2004, MNRAS, 347,246

Reid, N. 2003, MNRAS, 342, 837

de la Reza, R., Jilinski, E., \& Ortega, V. G. 2006, AJ, 131, 2609

Roberts Jr., W. W. 1969, ApJ, 158, 123

Rucinski, S. M., \& Krautter, J. 1983, A\&A, 121, 217

Sartori, M. J., Lépine, J. R. D., \& Dias, W. S. 2001, in Young stars near Earth: Progress and Prospects, ed. R. Jayawardhana, \& T. P. Greene, ASP Conf. Ser., 244,98

Sartori, M. J., Lépine, J. R. D., \& Dias, W. S. 2003, A\&A, 404, 913 [S03]

Sfeir, D. M., Lallement, R., Crifo, F., \& Welsh, B. Y. 1999, A\&A, 346, 785

Shu, F. H., Milione, V., Gebel, W., et al. 1972, ApJ, 173, 557

Smith, B. A., \& Terrile, R. J. 1984, Science, 226, 1421

Smith, R. K., \& Cox, D. P. 2001, ApJS, 134, 283

Snowden, S. L., Cox, D. P., McCammon, D., \& Sanders, W. T. 1990, ApJ, 354, 211

Snowden, S. L., Egger, R., Finkbeiner, D. P., Freyberg, M. J., \& Plucinsky, P. P. 1998, ApJ, 493, 715
Soderblom, D. R., King, J. R., Siess, L., et al. 1998, ApJ, 498, 385 Song, I., Zuckerman, B., \& Bessell, M. S. 2003, ApJ, 599, 342

Song, I., Zuckerman, B., \& Bessell, M. S. 2004, ApJ, 600, 1016

Stauffer, J. R., Schultz, G., \& Kirkpatrick, J. D. 1998, ApJS, 499, 199

Stelzer, B., \& Neuhaüser, R. 2000, A\&A, 361, 581

Sterzik, M., \& Durisen, R. 1995, A\&A, 304, L9

Sterzik, M., Alcalá, J. M., Covino, E., \& Petr, M. G. 1999, A\&A, 346, L41

Taylor, K. N. R., \& Storey, J. W. V. 1984, MNRAS, 209, 5P

Terranegra, L., Morale, F., Spagna, A., Massone, G., \& Lattanzi, M. G. 1999, A\&A, 341, L79

Torra, J., Fernández, D., \& Figueras, F. 2000, A\&A, 359, 82

Torres, C. A. O., da Silva, L., Quast, G., de la Reza, R., \& Jilinski, E. 2000, AJ, 120,1410

Torres, C. A. O., Quast, G. R., de la Reza, R., da Silva, L., \& Melo, C. H. F. 2001, in Young stars near Earth: Progress and Prospects, ed. R. Jayawardhana, \& T. P. Greene, ASP Conf. Ser., 244, 43

Torres, G., Guenther, E. W., Marschall, L. A., et al. 2003, AJ, 125, 825

Vanhala, H. A., \& Cameron, A. G. W. 1998, ApJ, 508, 291

Voges, W., Aschenbach, B., Boller, T., et al. 2000, Max-Planck-Institut für extraterrestrische Physik, Garching, http://www.xray.mpg.de/rosat/ survey/rass. fsc/

Webb, R. A., Zuckerman, B., Platais, I., et al. 1999, ApJ, 512, L63

Weiler, K. W., \& Sramek, R. A. 1998, ARA\&A, 26, 295

Weinberger, A. J., Rich, R. M., Becklin, E. E., Zuckerman, B., \& Matthews, K. 2000, ApJ, 544, 937

Weinberger, A. J., Becklin, E. E., Zuckerman, B., Schneider, G., \& Silverstone, M. D. 2001, in Young stars near Earth: Progress and Prospects, ed. R. Jayawardhana, \& T. P. Greene, ASP Conf. Ser., 244, 75

Weintraub, D. A., Kastner, J. H., Hines, D. C., \& Sahai, R. 2000, ApJ, 531, 401

Welsh, B. Y., Craig, N., Vedder, P. W., \& Vallerga, J. V. 1994, ApJ, 437, 638

Welsh, B. Y., Sfeir, D. M., Sirk, M. M., \& Lallement, R. 1999, A\&A, 352, 308

Wilking, B. A., Taylor, K. N. R., \& Storey, J. W. V. 1986, AJ, 92, 103

Wilking, B. A., Greene, T. P., Lada, C. J., Meyer, M. R., \& Young, E. T. 1992 , ApJ, 397, 520

Wilking, B. A., McCaughrean, M. J., Burton, M. G., et al. 1997, AJ, 114, 2029

Yuan, C., \& Grosbøl, P. 1981, ApJ, 243, 432

de Zeeuw, P. T., Hoogerwerf, R., Bruijne, J. H. J., Brown, A. G. A., \& Blaauw, A. 1999, AJ, 117, 354 [Z99]

Zuckerman, B., \& Song, I. 2004, ARA\&A, 42, 685

Zuckerman, B., \& Webb, R. A. 2000, ApJ, 535, 959

Zuckerman, B., Song, I., Bessell, M. S., \& Webb, R. A. 2001a, ApJ, 562, L87

Zuckerman, B., Song, I., \& Webb, R. A. 2001b, ApJ, 559, 388

Zuckerman, B., Webb, R. A., Schwartz, M., \& Becklin, E. E. 2001c, ApJ, 549, L233

Zuckerman, B., Song, I., \& Bessell, M. S. 2004, ApJ, 613, L65 


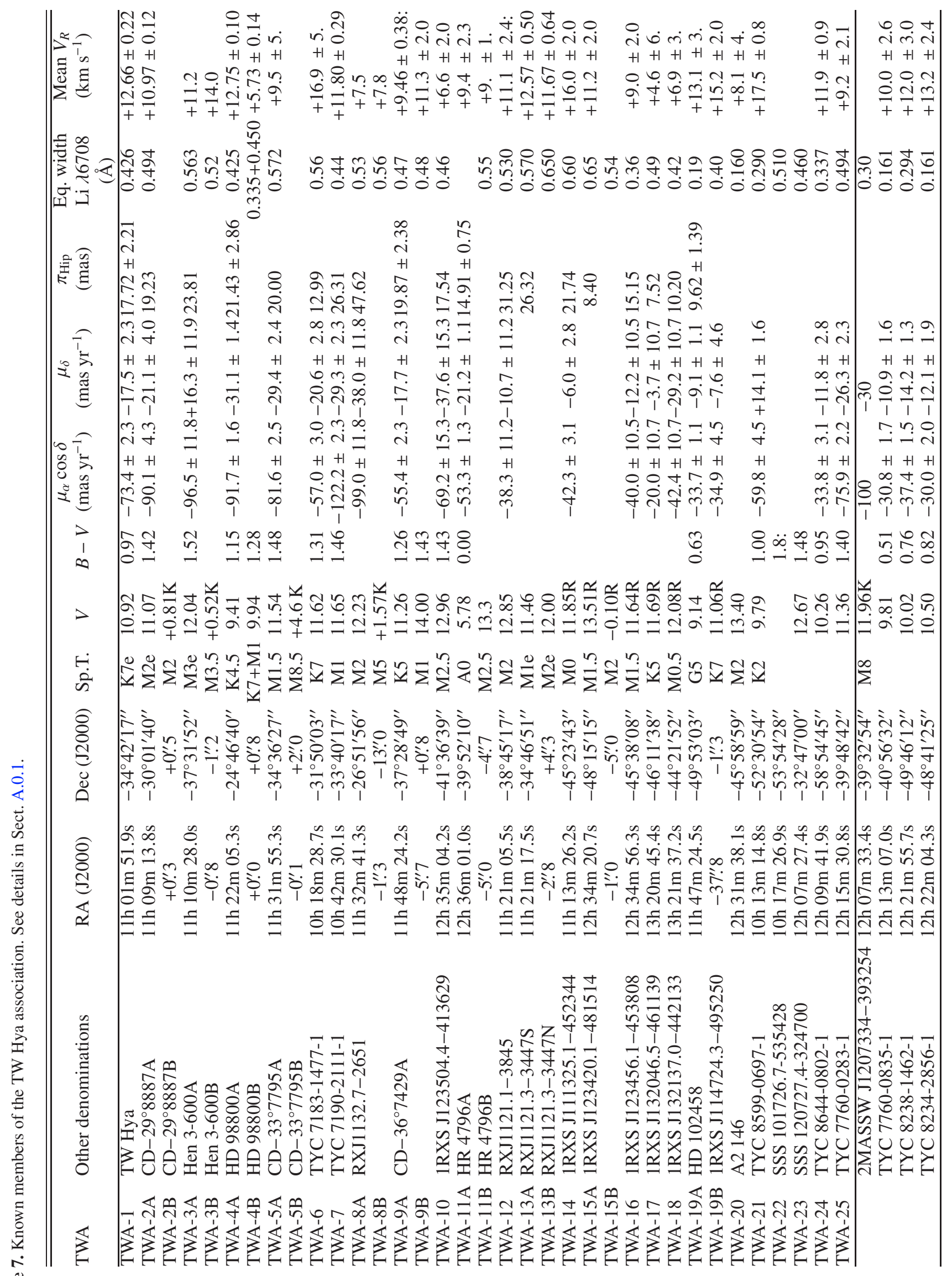




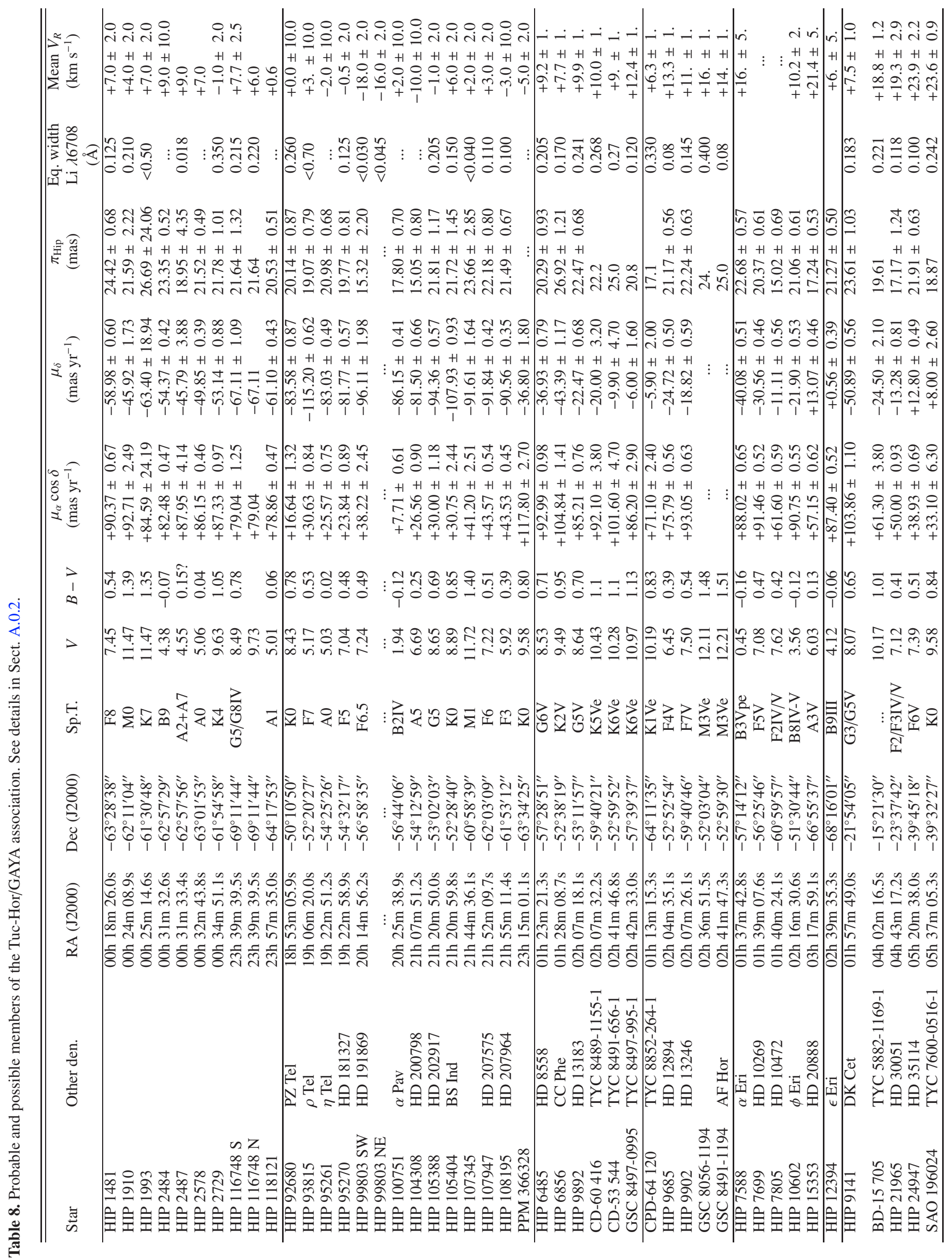


D. Fernández et al.: On the kinematic evolution of young local associations and the Scorpius-Centaurus complex, Online Material $p 3$

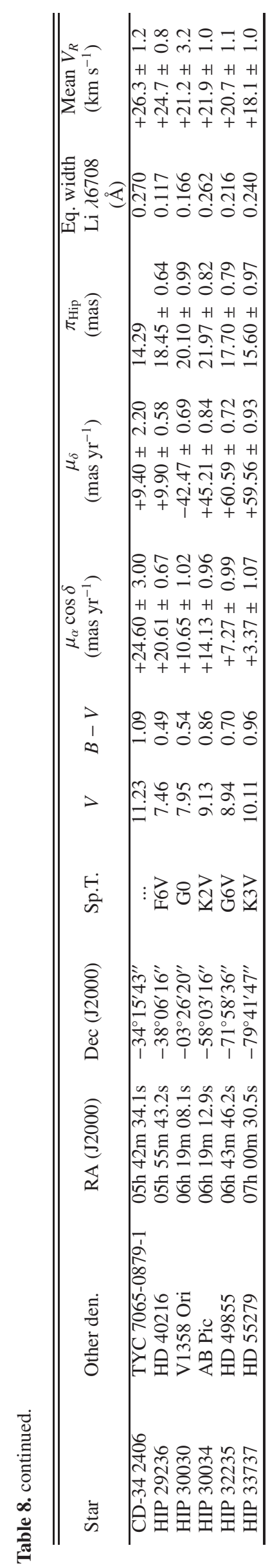


D. Fernández et al.: On the kinematic evolution of young local associations and the Scorpius-Centaurus complex, Online Material p 4

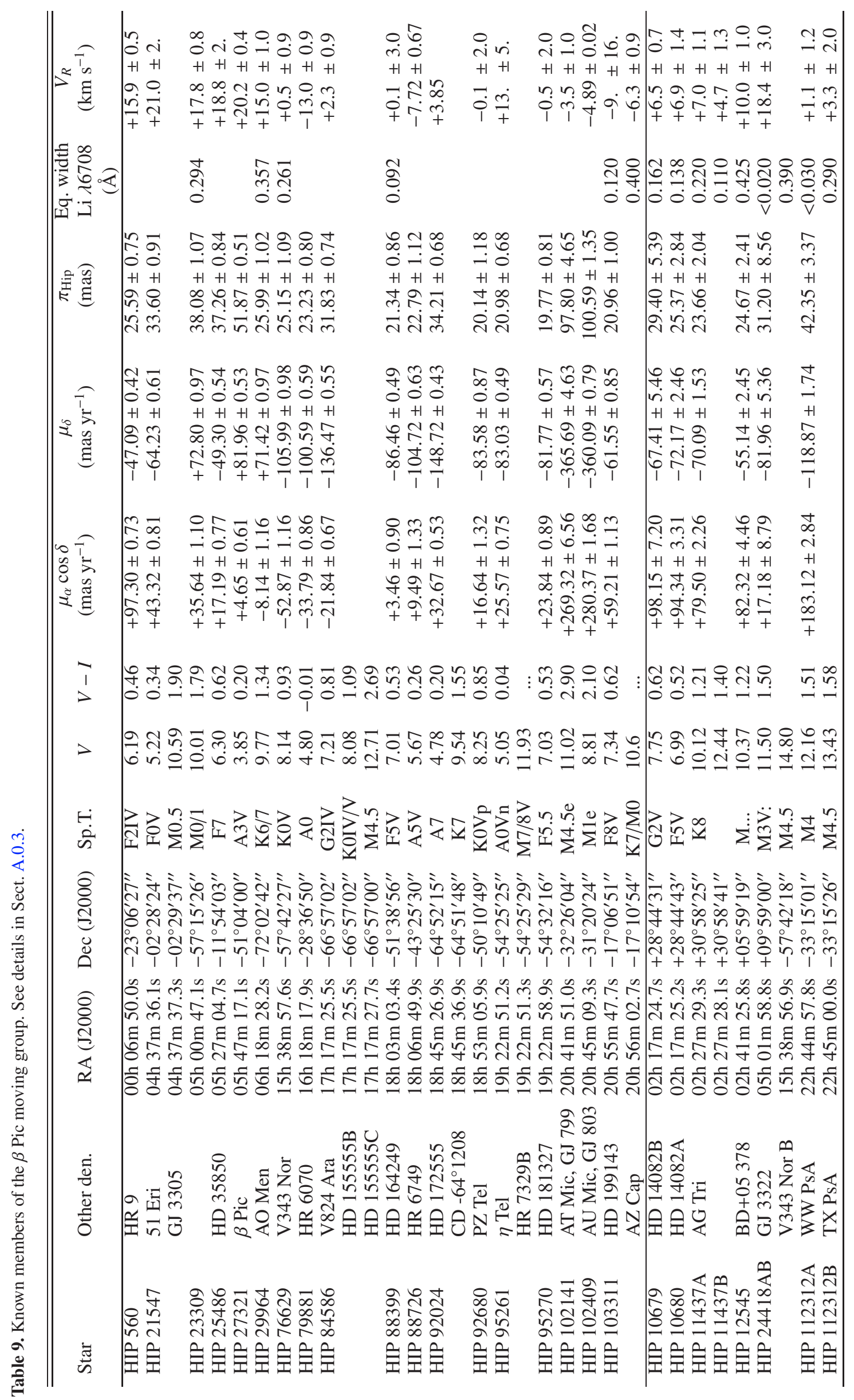


D. Fernández et al.: On the kinematic evolution of young local associations and the Scorpius-Centaurus complex, Online Material p 5

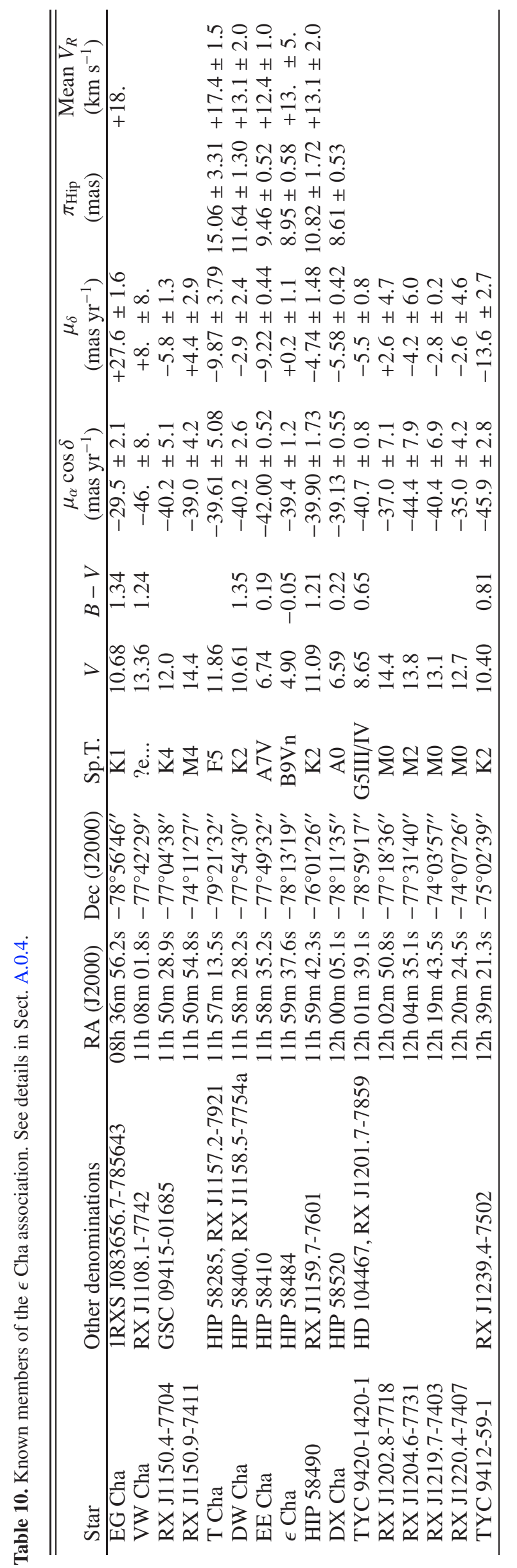


D. Fernández et al.: On the kinematic evolution of young local associations and the Scorpius-Centaurus complex, Online Material $p 6$

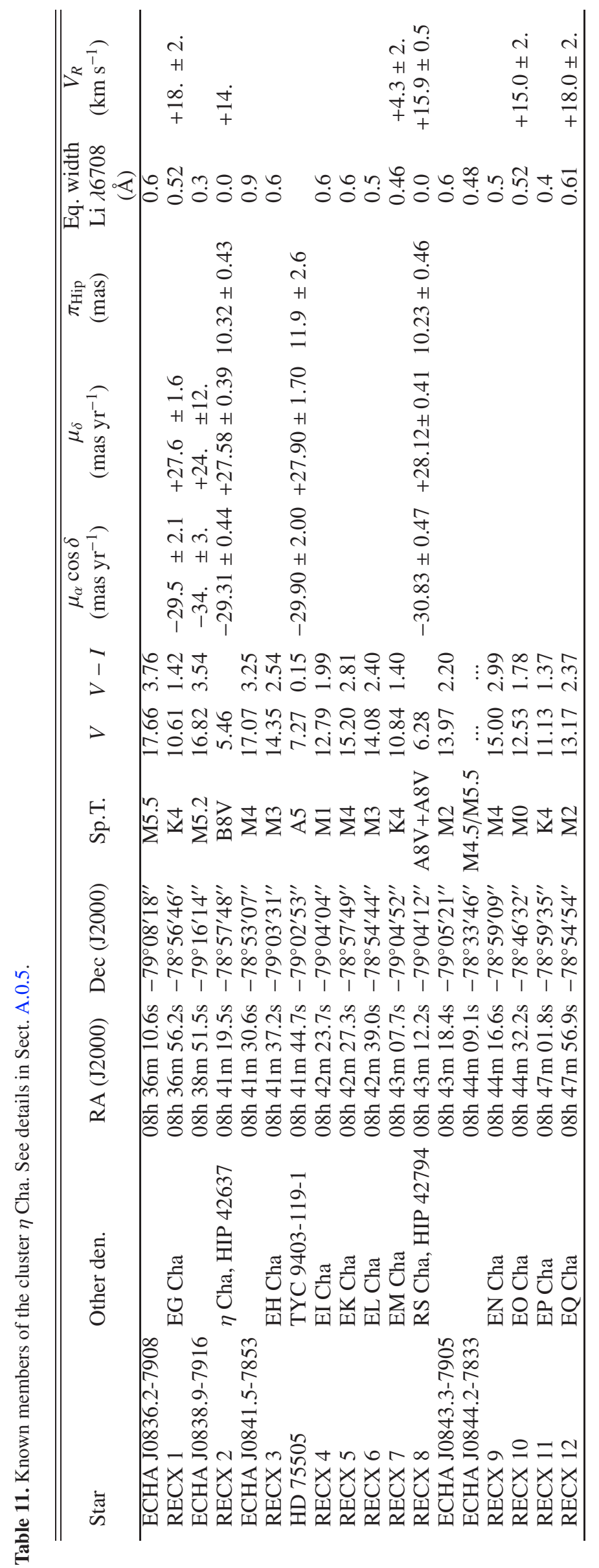




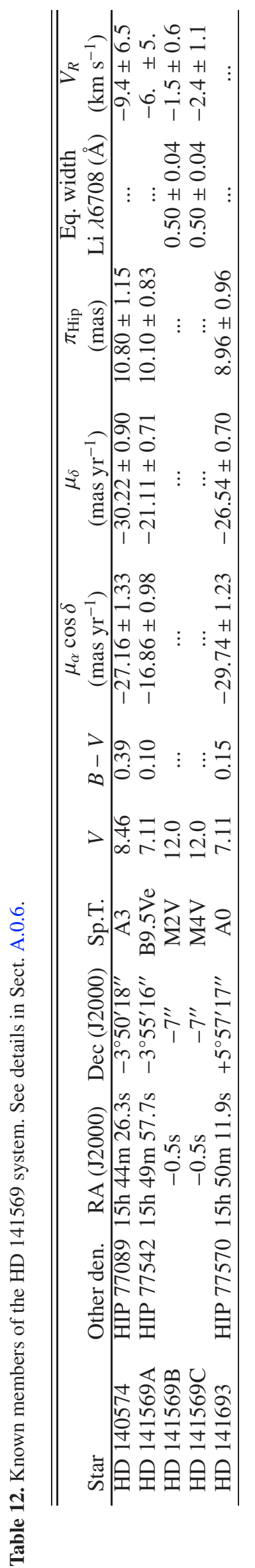




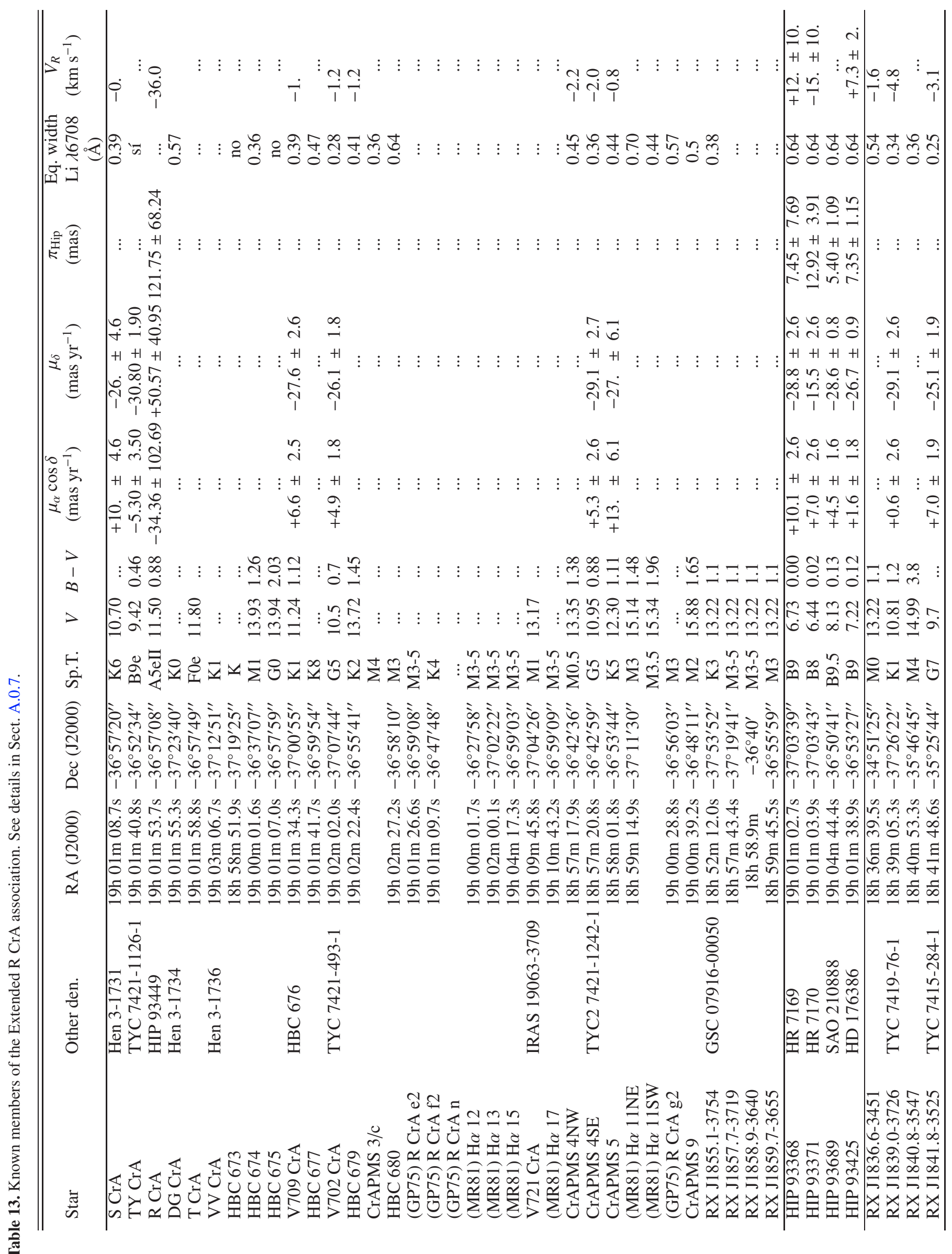


D. Fernández et al.: On the kinematic evolution of young local associations and the Scorpius-Centaurus complex, Online Material $p 9$

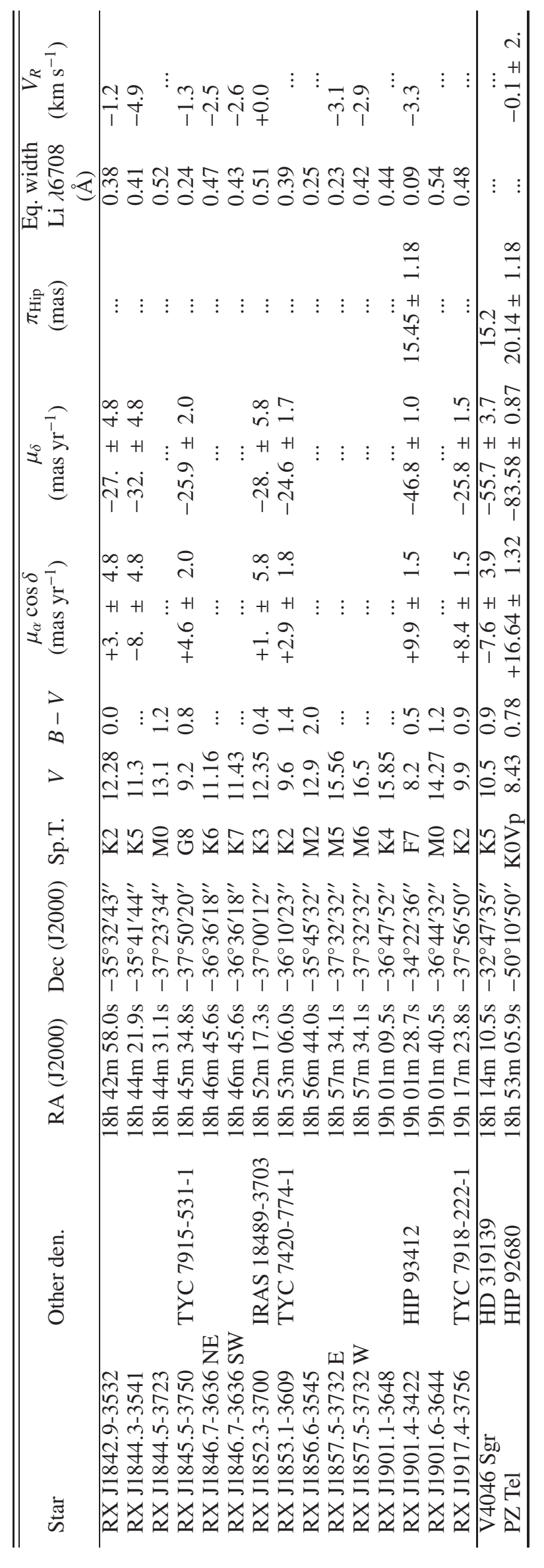




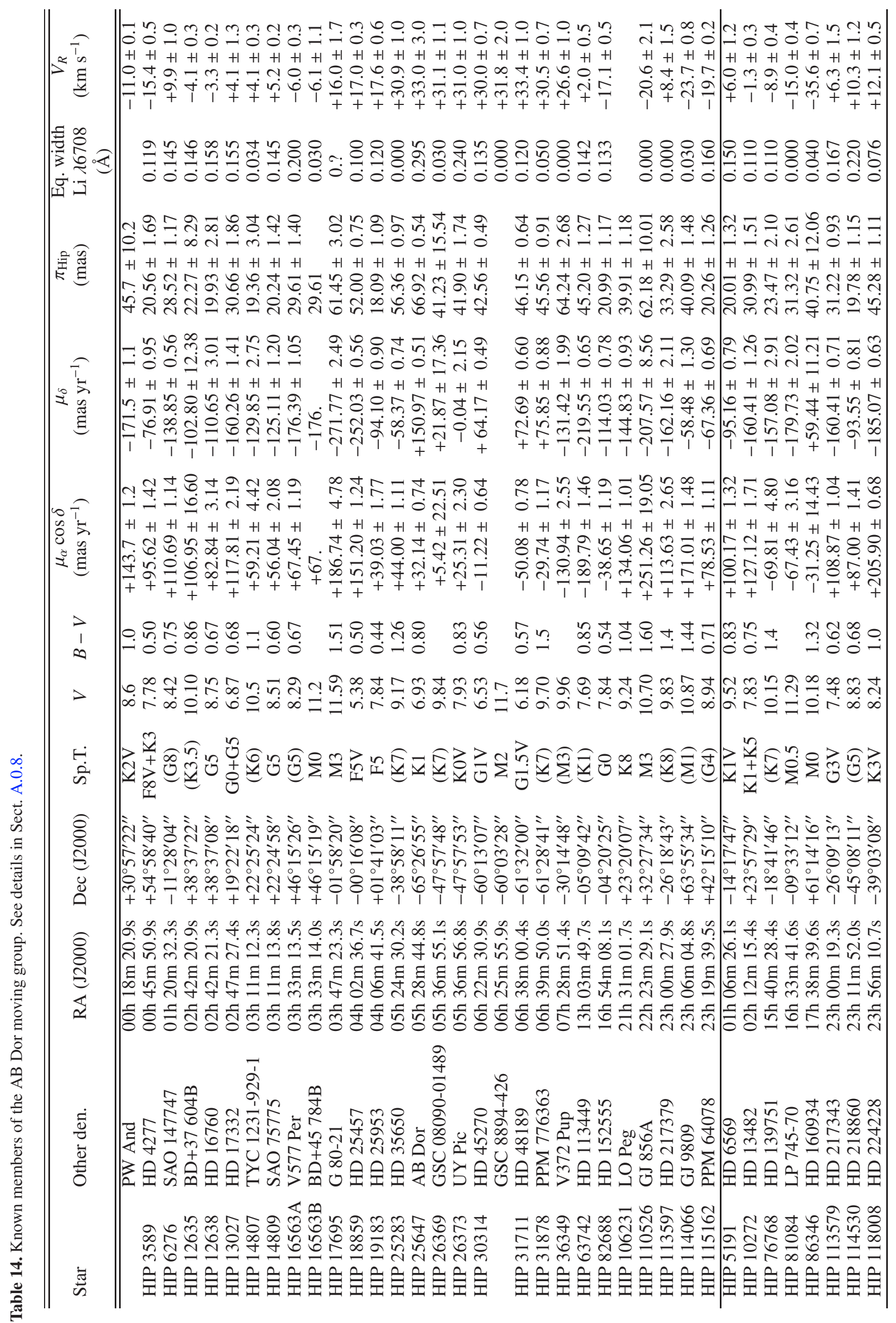

